\title{
Dirac approach to constrained submanifolds in a double loop group: From Wess-Zumino-Novikov-Witten to Poisson-Lie $\sigma$-model
}

\author{
H. Montani ${ }^{1, a)}$ and M. Zuccalli ${ }^{2, b)}$ \\ ${ }^{1}$ CONICET and Departamento de Ciencias Exactas y Naturales, \\ Universidad Nacional de la Patagonia Austral, (9011)Caleta Olivia, Argentina \\ ${ }^{2}$ Departamento de Matemática, Universidad Nacional de La Plata, \\ Calle 50 Esq. 115 (1900) La Plata, Argentina
}

(Received 7 January 2014; accepted 30 August 2014; published online 16 September 2014)

\begin{abstract}
We study the restriction to a family of second class constrained submanifolds in the cotangent bundle of a double Lie group equipped with a 2-cocycle extended symplectic form to build the corresponding Dirac brackets. It is shown that, for 2cocycle vanishing on each isotropic subspace of the associated Manin triple, the Dirac bracket contains no traces of the cocycle. We also investigate the restriction of the left translation action of the double Lie group on its cotangent bundle, where it fails to be a canonical transformation. However, the Hamiltonian symmetry is restored on some special submanifolds. The main application is to loop groups, showing that a WZNW-type model on the double Lie group with a quadratic Hamilton function in the momentum maps associated with the left translation action on the cotangent bundle with the canonical symplectic form, restricts to a collective system on some special submanifolds. There, the Lagrangian version coincides with the so-called Poisson-Lie $\sigma$-model. @ 2014 AIP Publishing LLC. [http://dx.doi.org/10.1063/1.4895465]
\end{abstract}

\section{INTRODUCTION}

Many relevant physical systems are modeled on Lie groups, taking the corresponding cotangent bundles as their phase spaces. Among the finite dimensional examples are the rigid bodies and their generalizations ${ }^{3}$ and, in infinite dimension, the sigma and WZNW models are outstanding field theories. Cotangent bundles are canonically symplectic manifolds and have rich structures underlying symmetries that involve their Lie algebras and their dual spaces. ${ }^{1,4,13,18}$ In some special situations, these structures straightforwardly lead to integrability. ${ }^{21}$

In the last decades, the dynamics of integrable systems has become more involved with Lie groups: it turns out that most of them are deeply related to Poisson-Lie groups, that is, Lie groups with a compatible Poisson structure..$^{10}$ A Poisson-Lie group has a dualPoisson-Lie group naturally associated, and the double Lie group built with this dual pair is, in some sense, a self-dual structure with a lot of nice properties enriching the framework of integrability. ${ }^{10,16,22}$

In Ref. 8, by regarding the cotangent bundle of this kind of double Lie group as a fibration on one of its factors, the Dirac method ${ }^{9}$ was developed for dealing with the restriction to the fibers of a dynamical system on the whole space. In fact, these fibers turn to be symplectic submanifolds of the cotangent bundle of the double Lie group equipped with the canonical symplectic form, turning the restriction to them in to a second class constraint problem.

Cotangent bundles of Lie groups equipped with the canonical symplectic structure are not enough to encode the plethora of systems modeled on Lie groups. For instance, the phase space of a sigma model with target space the group manifold $G$ is the cotangent bundle $T^{*} L G$ of the loop group

\footnotetext{
a)E-mail: hmontani@uaco.unpa.edu.ar

b)E-mail: marcezuccalli@gmail.com
} 
$L G$ with the canonical symplectic form $\omega_{0}$, and the dynamics is determined by the selection of the Hamilton function. On the other hand, the WZNW model shares the same configuration space, but it cannot be obtained from this phase space: no Hamilton function leading to Hamilton equations equivalent to the WZNW ones can be found. In fact, it was shown in Ref. 11 that the addition of Wess-Zumino term, the topological term, to the action of the sigma model amounts to a modification of the canonical Poisson brackets. Its symplectic counterpart is exhaustively studied in Ref. 14, where a cocycle extension of the canonical symplectic form $\omega_{0}$ is considered in combination with the Marsden-Weinstein reduction by stages procedure in order to recover the WZNW equation of motion. ${ }^{19}$

In this work, we adapt the scheme developed in Ref. 8 to the case where the initial phase space is the cotangent bundle $T^{*} G$ of a double Poisson-Lie group $G=G_{+} G_{-}$equipped with the symplectic form $\omega_{c}$ obtained by modifying the canonical one by adding a 2-cocycle $c: \mathfrak{g} \otimes \mathfrak{g} \longrightarrow \mathbb{R}$ on the Lie algebra $\mathfrak{g}$ of the double Lie group $G$. So, Hamiltonian systems on this symplectic manifold are of the WZNW-type in the sense that their Lagrangian counterpart exhibits the topological WZ-term. We consider dynamical systems on the fiber of the fibration $T^{*} G \longrightarrow T^{*} G_{-}$that are symplectic submanifolds of $\left(T^{*} G, \omega_{c}\right)$, so they can be addressed via the Dirac method for second class constraints as in Ref. 8. Thus, we build the Dirac brackets to describe dynamical systems on these constrained submanifolds in terms of the algebra of functions on the whole space $T^{*} G$. We investigate how the left action of the group $G$ on itself, lifted to its cotangent bundle, restricts to the fibers and becomes a symmetry of these phase subspaces for some special fibers. This scheme becomes very useful when applied to the specific case of loop groups, where amazingly the Dirac brackets lose the cocycle contributions. We work out the restriction of a quadratic Hamiltonian on $T^{*} G$ that becomes collective on the same fibers where the left translation action turns to be a symmetry, recovering a Poisson-Lie $\sigma$-model on each of these fibers. All these facts bring the subject into the realm of Poisson-Lie T-duality, ${ }^{15}$ turning this machinery very useful for working on the Hamiltonian approach to it. ${ }^{6,7}$

This work is organized as follows: we divided it into two main parts. In Part I, we concentrate on developing the Dirac machinery and symmetry issues for the 2-cocycle extended symplectic form on $T^{*} G$. Thus, in Sec. II A we describe the phase space on a double Poisson-Lie group and build the fibration of constrain submanifolds. In Sec. II B, we adapt the scheme developed in Ref. 8 to the case where the canonical symplectic form is modified by adding a 2-cocycle. Section IIC is devoted to apply the construction of Sec. II B to the case of loop groups. In Sec. II D, we discuss the left translation symmetry including the action by left translation of the centrally extended group. Section IIE introduces the Hamilton equations for the whole and the constrained spaces, and describes some properties of the collective dynamics. In Part II, we develop the main application of the results of Part I, mainly addressed to the loop group context. So, in Sec. III A we address a Hamiltonian model that restricts to a collective one. In Secs. III B and IIIC we retrieve the Lagrange equations, explicitly introducing the loop groups stage, making contact with the so-called Poisson-Lie $\sigma$-models. Finally, in Sec. IV some conclusions are summarized.

\section{PART I}

\section{PHASE SPACES ON DOUBLE LIE GROUPS AND CONSTRAINED SYSTEMS}

In this first part, we study the fibration $\Psi: G \times \mathfrak{g}^{*} \longrightarrow G_{-} \times \mathfrak{g}_{-}^{*}$, for a double Lie group $G=G_{+} G_{-}$, as the phase space of systems constrained to the fibers $\Psi^{-1}\left(g_{-}, \eta_{-}\right)$. We adapt the Dirac's machinery developed in Ref. 8 to the framework of $G \times \mathfrak{g}^{*}$ equipped with the 2-cocycle extended symplectic form, pointing to the loop groups stage. We also address the left translation action of $G$ on $G \times \mathfrak{g}^{*}$ and its restriction to $\Psi^{-1}\left(g_{-}, \eta_{-}\right)$, finding out the fibers on which it turns in to a phase space symmetry with Ad-equivariant momentum maps. Collective dynamics is then possible on some fibers, so we study its properties in the current framework.

\section{A. The fibration $G \times \mathfrak{g}^{*} \longrightarrow G_{-} \times \mathfrak{g}_{-}^{*}$}

Let us describe the framework for the main developments in this work (we follow the definitions and notations in Ref. 16). Let $\left(\mathfrak{g}, \mathfrak{g}_{+}, \mathfrak{g}_{-}\right)$be Manin triple, that is, $\mathfrak{g}, \mathfrak{g}_{+}, \mathfrak{g}_{-}$are Lie algebras such that 
$\mathfrak{g}=\mathfrak{g}_{+} \oplus \mathfrak{g}_{-}$as a vector space, and $\mathfrak{g}$ is equipped with an ad-invariant nondegenerate symmetric bilinear form $(,)_{\mathfrak{g}}$ turning $\mathfrak{g}_{+}, \mathfrak{g}_{-}$into isotropic subspaces. Thus, $\mathfrak{g}$ is a double Lie algebraand $\mathfrak{g}_{+}, \mathfrak{g}_{-}$ are Lie bialgebras, a couple of dual Lie bialgebras. The associated simply connected Lie group $G$, $G_{+}, G_{-}$form a double Lie group $G=G_{+} G_{-}$, with $G_{+}$and $G_{-}$being Poisson-Lie groups. Let us denote the corresponding projectors $\Pi_{G_{ \pm}}: G \longrightarrow G_{ \pm}, \Pi_{\mathfrak{g}_{ \pm}}: \mathfrak{g} \longrightarrow \mathfrak{g}_{ \pm}$and $\Pi_{\mathfrak{g}_{ \pm}^{*}}: \mathfrak{g}^{*} \longrightarrow \mathfrak{g}_{ \pm}^{*}$, which for short we frequently denote as $g_{ \pm}=\Pi_{G_{ \pm}} g, X_{ \pm}=\Pi_{\mathfrak{g}_{ \pm}} X$ and $\eta_{ \pm}=\Pi_{\mathfrak{g}_{ \pm}^{*}} \eta$. The factorization of elements $g_{-} g_{+} \in G$ is denoted as

$$
g_{+}^{h_{-}}:=\Pi_{G_{+}}\left(h_{-} g_{+}\right), g_{-}^{h_{+}}:=\Pi_{G_{-}}\left(h_{-} g_{+}\right) .
$$

Indeed, the maps

$$
G_{\mp} \times G_{ \pm} \longrightarrow G_{ \pm} \quad / \quad\left(h_{\mp}, g_{ \pm}\right) \mapsto \Pi_{G_{ \pm}}\left(h_{\mp} g_{ \pm}\right)=g_{ \pm}^{h_{\mp}}
$$

amount to be crossed actions between the factors, the so-called dressing actions. ${ }^{16,22}$ The infinitesimal generator of the dressing action of $G_{-}$on $G_{+}$at the point $g_{+} \in G_{+}$gives rise to the antihomomorphism of Lie algebras $X_{-} \in \mathfrak{g}_{-} \mapsto g_{+}^{X_{-}} \in T_{g_{+}} G_{+}$, such that for $X_{-}, Y_{-} \in \mathfrak{g}_{-}$, $\left[g_{+}^{X_{-}}, g_{+}^{Y_{-}}\right]=-g_{+}^{\left[X_{-}, Y_{-}\right]_{\mathfrak{g}_{-}}}$.

Let $\psi$ be the identification $\mathfrak{g} \longrightarrow \mathfrak{g}^{*}$ provided by the nondegenerate bilinear form, and $\bar{\psi}$ denote its inverse, then the crossed adjoint actions are

$$
\left\{\begin{array}{l}
\operatorname{Ad}_{h_{+}^{-1}}^{G} X_{-}=h_{+}^{-1} h_{+}^{X}+\bar{\psi}\left(A d_{h_{+}}^{*} \psi\left(X_{-}\right)\right) \\
\operatorname{Ad}_{h_{-}}^{G} X_{+}=h_{-}^{X_{+}} h_{-}^{-1}+\bar{\psi}\left(A d_{h_{-}^{-1}}^{*} \psi\left(X_{+}\right)\right),
\end{array}\right.
$$

where $A d^{*}$ stands for the dual of the adjoint action of each group factor on the dual of its Lie algebra (it relates to the coadjoint action $A d^{\#}$ as $A d_{h_{+}}^{\#}:=A d_{h_{+}^{-1}}^{*}$ ). This expression allows us to write the infinitesimal generators as $g_{+}^{X_{-}}=g_{+}\left(\Pi_{\mathfrak{g}_{+}} \operatorname{Ad}_{g_{+}^{-1}}^{G} X_{-}\right)$and $g_{-}^{X_{+}}=\left(\Pi_{\mathfrak{g}_{-}} \operatorname{Ad}_{g_{-}}^{G} X_{+}\right) g_{-}$.

The starting point of our developments is the cotangent bundle of the double Lie group $G=$ $G_{+} G_{-}$, realized as $G \times \mathfrak{g}^{*}$ by using the left translation isomorphism, and the fibration is by the surjective submersion

$$
\begin{array}{r}
\Psi: G \times \mathfrak{g}^{*} \longrightarrow G_{-} \times \mathfrak{g}_{-}^{*} \\
(g, \eta) \longmapsto\left(g_{-}, \eta_{-}\right) .
\end{array}
$$

Let us name $\mathcal{N}\left(g_{-}, \eta_{-}\right)$the fiber on $\left(g_{-}, \eta_{-}\right)$, then

$$
\mathcal{N}\left(g_{-}, \eta_{-}\right):=\Psi^{-1}\left(g_{-}, \eta_{-}\right)=\left\{\left(g_{+} g_{-}, \eta_{+}+\eta_{-}\right) / g_{+} \in G_{+}, \eta_{+} \in \mathfrak{g}_{+}^{*}\right\} .
$$

The differential $\Psi_{*}$ of the map $\Psi: G \times \mathfrak{g}^{*} \longrightarrow G_{-} \times \mathfrak{g}_{-}^{*}$ can be obtained from

$$
g^{-1} \dot{g}=A d_{g_{-}^{-1}}^{G} g_{+}^{-1} \dot{g}_{+}+g_{-}^{-1} \dot{g}_{-}
$$

then,

$$
\left.\Psi_{*}(g X, \xi)\right|_{(g, \eta)}=\left(\left(\Pi_{\mathfrak{g}_{-}} \operatorname{Ad}_{g_{-}}^{G} X_{+}\right) g_{-}+g_{-} X_{-}, \xi_{-}\right)_{\left(g_{-}, \eta_{-}\right)} .
$$

The kernel of $\Psi_{*}$ coincides with $T \mathcal{N}\left(g_{-}, \eta_{-}\right)$, and it can be explicitly described as

$$
\left.\operatorname{ker} \Psi_{*}\right|_{(g, \eta)}=\left\{\left(g_{+}\left(\bar{\psi}\left(A d_{g_{-}^{-1}}^{*} \psi\left(X_{+}\right)\right)\right) g_{-}, \xi_{+}\right) /\left(X_{+}, \xi_{+}\right) \in \mathfrak{g}_{+} \oplus \mathfrak{g}_{+}^{*}\right\} .
$$

The Dirac method is built from the annihilator of $\left.\operatorname{ker} \Psi_{*}\right|_{(g, \eta)}$, which is the pullback of the cotangent bundle of the base space $G_{-} \times \mathfrak{g}_{-}^{*}$.

\section{B. Centrally extended symplectic structures and the Dirac method}

In this section, we adapt the Dirac bracket construction developed in Ref. 8 to the case where a canonical symplectic form on $G \times \mathfrak{g}^{*}$ is modified by adding an $\mathbb{R}$-valued 2 -cocycle on $\mathfrak{g}$ and we write the Dirac bracket on the submanifolds $\mathcal{N}_{c}\left(g_{-}, \eta_{-}\right)$. 
Let $C: G \longrightarrow \mathfrak{g}^{*}$ be a coadjoint 1-cocycle, that is, for $g, h \in G$ it satisfies

$$
C(g h)=\operatorname{Ad}_{g^{-1}}^{G *} C(h)+C(g), \forall g, h \in G .
$$

By considering $\hat{c}=-\left.d C\right|_{e}: \mathfrak{g} \longrightarrow \mathfrak{g}^{*}$, the 1-cocycle $C$ defines the application $c: \mathfrak{g} \otimes \mathfrak{g} \longrightarrow \mathbb{R}$ given by

$$
c(X, Y):=\langle\hat{c}(X), Y\rangle \text {. }
$$

It is easy to see that $c$ is bilinear, antisymmetric and verifies the Jacobi identity. Then, $c$ is an $\mathbb{R}$-valued 2 -cocycle on $\mathfrak{g}$ that satisfies the following condition:

$$
c\left(A d_{g} X, A d_{g} Y\right)=c(X, Y)+\left\langle C\left(g^{-1}\right),[X, Y]\right\rangle \text {. }
$$

So, let us consider the canonical symplectic form $\omega_{\circ}$ in $T^{*} G$. Then, by adding $c$ one defines a new symplectic form on $T^{*} G$ given by

$$
\begin{aligned}
& \left\langle\omega_{c},(v, \rho) \otimes(w, \xi)\right\rangle_{(g, \eta)} \\
:= & \left\langle\rho, g^{-1} w\right\rangle+\left\langle\xi, g^{-1} v\right\rangle+\left\langle\eta,\left[g^{-1} v, g^{-1} w\right]\right\rangle+c\left(v g^{-1}, w g^{-1}\right)
\end{aligned}
$$

for $(v, \rho),(w, \xi) \in T_{(g, \eta)}^{*} G$.

The Hamiltonian vector field of a function $\mathcal{F}: G \times \mathfrak{g}^{*} \longrightarrow \mathbb{R}$ is

$$
V_{\mathcal{F}}(g, \eta)=\left(g \delta \mathcal{F}, a d_{\delta \mathcal{F}}^{*} \eta-g \mathbf{d} \mathcal{F}+\operatorname{Ad}_{g}^{G *} \hat{c}\left(A d_{g}^{G} \delta \mathcal{F}\right)\right)
$$

for $(g, \eta) \in G \times \mathfrak{g}^{*}$, and the associated Poisson bracket is

$$
\{\mathcal{F}, \mathcal{G}\}_{c}(g, \eta)=\langle\delta \mathcal{G}, g \mathbf{d} \mathcal{F}\rangle-\langle\mathbf{d} \mathcal{G}, g \delta \mathcal{F}\rangle-\left\langle\eta+C\left(g^{-1}\right),[\delta \mathcal{F}, \delta \mathcal{G}]\right\rangle-c(\delta \mathcal{F}, \delta \mathcal{G})
$$

for $\mathcal{F}, \mathcal{G} \in C^{\infty}\left(G \times \mathfrak{g}^{*}\right)$.

Let us now proceed to adapt the Dirac method to the restrictions to the fibers $\mathcal{N}\left(g_{-}, \eta_{-}\right)$when the centrally extended symplectic form on $T^{*} G$ is considered.

Proposition: $\left(\mathcal{N}\left(g_{-}, \eta_{-}\right), \tilde{\omega}_{c}\right)$, where $\tilde{\omega}_{c}$ is the restriction to $\mathcal{N}\left(g_{-}, \eta_{-}\right)$of the centrally extended canonical symplectic form $\omega_{c}$ on $G \times \mathfrak{g}^{*}$, is a symplectic manifold.

Proof: Let us observe that the restriction of $\omega_{c}$ to the kernel of $\Psi_{*}$ is given by

$$
\begin{aligned}
& \left\langle\omega_{c},\left(g_{+}\left(\bar{\psi}\left(A d_{g_{-}^{-1}}^{*} \psi\left(X_{+}\right)\right)\right) g_{-}, \xi_{+}\right) \otimes\left(g_{+}\left(\bar{\psi}\left(A d_{g_{-}^{-1}}^{*} \psi\left(Y_{+}\right)\right)\right) g_{-}, \lambda_{+}\right)\right\rangle_{(g, \eta)} \\
= & \left\langle\omega,\left(g_{+}\left(\bar{\psi}\left(A d_{g_{-}^{-1}}^{*} \psi\left(X_{+}\right)\right)\right) g_{-}, \xi_{+}\right) \otimes\left(g_{+}\left(\bar{\psi}\left(A d_{g_{-}^{-1}}^{*} \psi\left(Y_{+}\right)\right)\right) g_{-}, \lambda_{+}\right)\right\rangle_{(g, \eta)} \\
& +c\left(g_{+}\left(\bar{\psi}\left(A d_{g_{-}^{-1}}^{*} \psi\left(X_{+}\right)\right)\right) g_{-}, g_{+}\left(\bar{\psi}\left(A d_{g_{-}^{-1}}^{*} \psi\left(Y_{+}\right)\right)\right) g_{-}\right)
\end{aligned}
$$

for $\left(g_{+}\left(\bar{\psi}\left(A d_{g_{-}^{-1}}^{*} \psi\left(X_{+}\right)\right)\right) g_{-}, \xi_{+}\right)$and $\left.\left(g_{+}\left(\bar{\psi}\left(A d_{g_{-}^{-1}}^{*} \psi\left(Y_{+}\right)\right)\right) g_{-}, \lambda_{+}\right) \in \operatorname{ker} \Psi_{*}\right|_{(g, \eta)}$. Then, is easy to see that there are no null vectors of $\omega_{c}$ on $T \Psi^{-1}\left(g_{-}, \eta_{-}\right)=\left.\operatorname{ker} \Psi_{*}\right|_{(g, \eta)}$.

Corollary: If $\left(T_{(g, \eta)} \mathcal{N}\left(g_{-}, \eta_{-}\right)\right)^{\perp \omega_{c}} \cap T_{(g, \eta)} \mathcal{N}\left(g_{-}, \eta_{-}\right)=\{0\}$, then $\Psi^{-1}\left(g_{-}, \eta_{-}\right)$is a second class constraint.

Here, $\left(T_{(g, \eta)} \mathcal{N}\left(g_{-}, \eta_{-}\right)\right)^{\perp \omega_{c}}$ denotes the orthogonal complement in relation to the symplectic structure $\omega_{c}$.

Now, we choose a basis for $T_{\left(g_{-}, \eta_{-}\right)}^{*}\left(G_{-} \times \mathfrak{g}_{-}^{*}\right) \cong \mathfrak{g}_{-}^{*} \oplus \mathfrak{g}_{-}$, where we use the left trivialization of $T_{\left(g_{-}, \eta_{-}\right)}^{*} G_{-}$. The basis $\left\{T_{a}\right\}$ of $\mathfrak{g}_{+}$and the basis $\left\{T^{a}\right\}$ of $\mathfrak{g}_{-}$provide a set of linearly independent 
1-forms on $G_{-} \times \mathfrak{g}_{-}^{*}$,

$$
\begin{aligned}
& \alpha_{a}=\left(L_{g_{-}}^{*} \psi\left(T_{a}\right), 0\right) \in T_{\left(g_{-}, \eta_{-}\right)}^{*} G_{-} \times \mathfrak{g}_{-}^{*}, \\
& \beta_{a}=\left(0, T^{a}\right) \in T_{\left(g_{-}, \eta_{-}\right)}^{*} G_{-} \times \mathfrak{g}_{-}^{*}
\end{aligned}
$$

such that for any $\left(v_{-}, \xi_{-}\right) \in T_{\left(g_{-}, \eta_{-}\right)} G_{-} \times \mathfrak{g}_{-}^{*}$,

$$
\begin{aligned}
& \left\langle\alpha_{a},\left(v_{-}, \xi_{-}\right)\right\rangle_{\left(g_{-}, \eta_{-}\right)}=\left(T_{a}, g_{-}^{-1} v_{-}\right)_{\mathfrak{g}} \\
& \left\langle\beta_{a},\left(v_{-}, \xi_{-}\right)\right\rangle_{\left(g_{-}, \eta_{-}\right)}=\left\langle\xi_{-}, T^{a}\right\rangle .
\end{aligned}
$$

The set of pullbacks $\left\{\Psi^{*} \alpha_{a}, \Psi^{*} \beta^{a}\right\}_{a}$ is linearly independent, with $T \mathcal{N}\left(g_{-}, \eta_{-}\right)$being their null distribution.

The associated Hamiltonian vector fields $V_{\Psi^{*} \alpha_{a}}$ and $V_{\Psi^{*} \beta^{a}}$ are

$$
\left\{\begin{array}{l}
V_{\Psi^{*} \alpha_{a}}(g, \eta)=\left(0,-\operatorname{Ad}_{g_{-}^{-1}}^{G} \Pi_{\mathfrak{g}_{+}} \operatorname{Ad}_{g_{-}}^{G} T_{a}\right) \\
V_{\Psi^{*} \beta^{a}}(g, \eta)=\left(g T^{a}, \operatorname{ad}_{T^{a}}^{\mathfrak{g} *} \eta-\lambda \operatorname{Ad}_{g}^{G *} \hat{c}\left(\operatorname{Ad}_{g}^{G} T^{a}\right)\right),
\end{array}\right.
$$

which allows us to calculate the Dirac matrix

$$
C(g, \eta)=\left(\begin{array}{cc}
C_{\Psi^{*} \alpha_{a}, \Psi^{*} \alpha_{b}}(g, \eta) & C_{\Psi^{*} \alpha_{a}, \Psi^{*} \beta^{b}}(g, \eta) \\
-C_{\Psi^{*} \alpha_{a}, \Psi^{*} \beta^{b}}(g, \eta) & C_{\Psi^{*} \beta^{a}, \Psi^{*} \beta^{b}}(g, \eta)
\end{array}\right)
$$

determined by the entries

$$
\begin{aligned}
& C_{\Psi^{*} \alpha_{a}, \Psi^{*} \alpha_{b}}(g, \eta)=\left\langle\Psi^{*} \alpha_{a}, V_{\Psi^{*} \alpha_{b}}\right\rangle_{(g, \eta)}=\left\langle\Psi^{*} \alpha_{a},\left(0, w_{\alpha_{b}}\right)\right\rangle_{(g, \eta)}=0 \\
& C_{\Psi^{*} \alpha_{a}, \Psi^{*} \beta^{b}}(g, \eta)=\left\langle\Psi^{*} \alpha_{a}, V_{\Psi^{*} \beta^{b}}\right\rangle_{(g, \eta)}=\delta_{a}^{b} \\
& C_{\Psi^{*} \beta^{a}, \Psi^{*} \beta^{b}}(g, \eta)=\left\langle\Psi^{*} \beta^{a}, V_{\Psi^{*} \beta^{b}}\right\rangle_{(g, \eta)}=\Omega_{c}^{a b}(g, \eta) .
\end{aligned}
$$

Here we write

$$
\Omega_{c}^{a b}(g, \eta):=-\left\langle C\left(g^{-1}\right)+\eta,\left[T^{a}, T^{b}\right]\right\rangle-c\left(T^{a}, T^{b}\right) .
$$

The Dirac matrix is then

$$
C(g, \eta)=\left(\begin{array}{cc}
0_{n \times n} & I_{n \times n} \\
-I_{n \times n} & \Omega_{c}(g, \eta)
\end{array}\right) .
$$

Now, we are ready to build up the Dirac brackets: for any couple of function $\mathcal{F}, \mathcal{G} \in$ $C^{\infty}\left(G \times \mathfrak{g}^{*}\right)$, the Dirac bracket gives the restriction of the Poisson bracket on $G \times \mathfrak{g}^{*}$ to the constrained submanifold $\mathcal{N}\left(g_{-}, \eta_{-}\right)$, and it is defined as

$$
\begin{aligned}
&\{\mathcal{F}, \mathcal{G}\}_{c}^{D}(g, \eta)=\{\mathcal{F}, \mathcal{G}\}_{c}(g, \eta) \\
&+\left\langle\eta+C\left(g^{-1}\right),\left[\Pi_{\mathfrak{g}_{-}}\{\mathcal{F}, \alpha\}, \Pi_{\mathfrak{g}_{-}}\{\alpha, \mathcal{G}\}\right]\right\rangle_{(g, \eta)} \\
&+\langle\{\mathcal{F}, \alpha\},\{\beta, \mathcal{G}\}\rangle-\langle\{\mathcal{F}, \beta\},\{\alpha, \mathcal{G}\}\rangle_{(g, \eta)} \\
&+c\left(\Pi_{\mathfrak{g}_{-}}\{\mathcal{F}, \alpha\}_{c}(g, \eta), \Pi_{\mathfrak{g}_{-}}\{\alpha, \mathcal{G}\}_{c}(g, \eta)\right)
\end{aligned}
$$

where

$$
\begin{aligned}
\Pi_{\mathfrak{g}_{-}}\{\mathcal{F}, \alpha\} & =\left\{\mathcal{F}, \alpha_{a}\right\}(g, \eta) T^{a}=-\operatorname{Ad}_{g_{-}^{-1}}^{G} \Pi_{\mathfrak{g}_{-}} \operatorname{Ad}_{g_{-}}^{G} \delta \mathcal{F} \\
\Pi_{\mathfrak{g}_{+}}\{\mathcal{F}, \beta\}(g, \eta) & =\Pi_{\mathfrak{g}_{+}}\left(g \mathbf{d} \mathcal{F}-\operatorname{ad}_{\delta \mathcal{F}}^{\mathfrak{g} *}\left(C\left(g^{-1}\right)+\eta\right)+c\left(T^{a}, \delta \mathcal{F}\right) T_{a}\right) .
\end{aligned}
$$

Thus, we have the following result. 
Proposition: The Dirac bracket on the submanifolds $\mathcal{N}_{c}\left(g_{-}, \eta_{-}\right)$for any couple of function $\mathcal{F}, \mathcal{G} \in C^{\infty}\left(G \times \mathfrak{g}^{*}\right)$ is

$$
\begin{aligned}
\{\mathcal{F}, \mathcal{G}\}_{c}^{D}(g, \eta)= & \left\langle g \mathbf{d} \mathcal{F}, \operatorname{Ad}_{g_{-}^{-1}}^{G} \Pi_{\mathfrak{g}_{+}} \operatorname{Ad}_{g_{-}}^{G} \delta \mathcal{G}\right\rangle-\left\langle g \mathbf{d} \mathcal{G}, \operatorname{Ad}_{g_{-}^{-1}}^{G} \Pi_{\mathfrak{g}_{+}} \operatorname{Ad}_{g_{-}}^{G} \delta \mathcal{F}\right\rangle \\
& -\left\langle\eta,\left[\operatorname{Ad}_{g_{-}^{-1}}^{G} \Pi_{\mathfrak{g}_{+}} \operatorname{Ad}_{g_{-}}^{G} \delta \mathcal{F}, \operatorname{Ad}_{g_{-}^{-1}}^{G} \Pi_{\mathfrak{g}_{+}} \operatorname{Ad}_{g_{-}}^{G} \delta \mathcal{G}\right]\right\rangle \\
& -\left\langle C\left(g_{+}^{-1}\right),\left[\Pi_{\mathfrak{g}_{+}} \operatorname{Ad}_{g_{-}}^{G} \delta \mathcal{F}, \Pi_{\mathfrak{g}_{+}} \operatorname{Ad}_{g_{-}}^{G} \delta \mathcal{G}\right]\right\rangle \\
& -c\left(\Pi_{\mathfrak{g}_{+}} \operatorname{Ad}_{g_{-}}^{G} \delta \mathcal{F}, \Pi_{\mathfrak{g}_{+}} \operatorname{Ad}_{g_{-}}^{G} \delta \mathcal{G}\right) .
\end{aligned}
$$

It is a nondegenerate bracket.

Remark I: In the particular case $\left(g_{-}, \eta_{-}\right)=(e, 0)$ we recover the cotangent bundle of $G_{+}$ equipped with the Poisson structure

$$
\begin{aligned}
\{\mathcal{F}, \mathcal{G}\}_{c}^{D}\left(g_{+}, \eta_{+}\right)= & \left\langle g \mathbf{d} \mathcal{F}, \Pi_{\mathfrak{g}_{+}} \delta \mathcal{G}\right\rangle-\left\langle g \mathbf{d} \mathcal{G}, \Pi_{\mathfrak{g}_{+}} \delta \mathcal{F}\right\rangle \\
& -\left\langle\eta+C\left(g_{+}^{-1}\right),\left[\Pi_{\mathfrak{g}_{+}} \delta \mathcal{F}, \Pi_{\mathfrak{g}_{+}} \delta \mathcal{G}\right]\right\rangle \\
& -c\left(\Pi_{\mathfrak{g}_{+}} \delta \mathcal{F}, \Pi_{\mathfrak{g}_{+}} \delta \mathcal{G}\right) .
\end{aligned}
$$

Remark II: Let us suppose that the restriction of the cocycle on $G$ to $G_{ \pm}$is such that

$$
\left.C\right|_{G_{ \pm}}: G_{ \pm} \longrightarrow \mathfrak{g}_{\mp}^{*}
$$

which in turn implies that

$$
\left.\hat{c}\right|_{\mathfrak{g}_{ \pm}}: \mathfrak{g}_{ \pm} \longrightarrow \mathfrak{g}_{\mp}^{*}
$$

then, for any couple of function $\mathcal{F}, \mathcal{G} \in C^{\infty}\left(G \times \mathfrak{g}^{*}\right)$, the Dirac bracket on $\mathcal{N}_{c}\left(g_{-}, \eta_{-}\right)$of Eq. (3) reduces to

$$
\begin{aligned}
\{\mathcal{F}, \mathcal{G}\}_{c}^{D}(g, \eta)= & \left\langle g \mathbf{d} \mathcal{F}, \operatorname{Ad}_{g_{-}^{-1}}^{G} \Pi_{\mathfrak{g}_{+}} \operatorname{Ad}_{g_{-}}^{G} \delta \mathcal{G}\right\rangle-\left\langle g \mathbf{d} \mathcal{G}, \operatorname{Ad}_{g_{-}^{-1}}^{G} \Pi_{\mathfrak{g}_{+}} \operatorname{Ad}_{g_{-}}^{G} \delta \mathcal{F}\right\rangle \\
& -\left\langle\eta,\left[\operatorname{Ad}_{g_{-}^{-1}}^{G} \Pi_{\mathfrak{g}_{+}} \operatorname{Ad}_{g_{-}}^{G} \delta \mathcal{F}, \operatorname{Ad}_{g_{-}^{-1}}^{G} \Pi_{\mathfrak{g}_{+}} A d_{g_{-}}^{G} \delta \mathcal{G}\right]\right\rangle,
\end{aligned}
$$

where there are no traces of the cocycle. Moreover, at $\left(g_{-}, \eta_{-}\right)=(e, 0)$ we recover the tangent bundle of $G_{+}$with the canonical bracket

$$
\begin{aligned}
\{\mathcal{F}, \mathcal{G}\}_{c}^{D}\left(g_{+}, \eta_{-}\right)= & \left\langle g \mathbf{d} \mathcal{F}, \Pi_{\mathfrak{g}_{+}} \delta \mathcal{G}\right\rangle-\left\langle g \mathbf{d} \mathcal{G}, \Pi_{\mathfrak{g}_{+}} \delta \mathcal{F}\right\rangle \\
& -\left\langle\eta,\left[\Pi_{\mathfrak{g}_{+}} \delta \mathcal{F}, \Pi_{\mathfrak{g}_{+}} \delta \mathcal{G}\right]\right\rangle .
\end{aligned}
$$

The Hamiltonian vector field in this case is

$$
V_{\mathcal{H}}(g, \eta)=\left(g\left(\operatorname{Ad}_{g_{-}^{-1}}^{G} \Pi_{\mathfrak{g}_{+}} \operatorname{Ad}_{g_{-}}^{G} \delta \mathcal{H}\right), \operatorname{Ad}_{g_{-}}^{G *} \Pi_{\mathfrak{g}_{+}^{*}} \operatorname{Ad}_{g_{-}^{-1}}^{G *}\left(\operatorname{ad}_{\operatorname{Ad}_{g_{-}^{-1}}^{G} \Pi_{\mathfrak{g}_{+}} \operatorname{Ad}_{g_{-}}^{G} \delta \mathcal{H}^{\prime}} \eta\right)\right) .
$$

\section{Loop groups}

Loop groups constitute the main application of the above construction, also considering their central extension. Thus, let $G=L H$ denote the set of maps from $S^{1}$ to the Lie group $H$, and $\mathfrak{g}=L \mathfrak{h}$ from $S^{1}$ to the Lie algebra $\mathfrak{h}$ of $H$. We assume that $\mathfrak{h}$ is equipped with a nondegenerate $\mathrm{Ad}^{H}$-invariant symmetric bilinear form $(,)_{\mathfrak{h}}$.

For $g \in G, g^{\prime}$ denotes the derivative in the loop parameter $s \in S^{1}$, and we write $v g^{-1}$ and $g^{-1} v$ for the right and left translation of any vector field $v \in T G$. Frequently, we work with the dense subset $L \mathfrak{h}^{*} \subset(L \mathfrak{h})^{*}$ instead of $(L \mathfrak{h})^{*}$, and we identify it with $L \mathfrak{h}$ through the map $\psi: L \mathfrak{h} \rightarrow L \mathfrak{h}^{*}$ 
provided by the bilinear form

$$
(,)_{\mathfrak{g}} \equiv \frac{1}{2 \pi} \int_{S^{1}}(,)_{\mathfrak{h}}
$$

on $\mathfrak{g}$. In this framework, the two cocycle $c_{\mathrm{k}}: \mathfrak{g} \times \mathfrak{g} \longrightarrow \mathbb{R}$ is given by the bilinear form $\Gamma_{\mathrm{k}}: \mathfrak{g} \times \mathfrak{g}$ $\rightarrow \mathbb{R},{ }^{20}$

$$
c_{\mathrm{k}}(X, Y) \equiv \Gamma_{\mathrm{k}}(X, Y)=\frac{\mathrm{k}}{2 \pi} \int_{S^{1}}\left(X(s), Y^{\prime}(s)\right)_{\mathfrak{h}} d s
$$

with $X(s), Y(s) \in \mathfrak{h}$. It is derived from the one cocycle $C_{\mathrm{k}}: G \rightarrow \mathfrak{g}^{*}$,

$$
C_{\mathrm{k}}(l)=\mathrm{k} \psi\left(l^{\prime} l^{-1}\right) .
$$

Note that it is a coadjoint cocycle

$$
C_{\mathrm{k}}(k l)=\operatorname{Ad}_{k^{-1}}^{G *} C_{\mathrm{k}}(l)+C_{\mathrm{k}}(k) .
$$

As above, we assume that $H=H_{+} H_{-}$with $H_{+}, H_{-}$are Lie subgroups of $H$, and $\mathfrak{h}=\mathfrak{h}_{+} \oplus \mathfrak{h}_{-}$, where $\mathfrak{h}_{+}, \mathfrak{h}_{-}$are Lie subalgebras of $\mathfrak{h}$. Moreover, we assume that the subspaces $\mathfrak{h}_{+}, \mathfrak{h}_{-}$are isotropic in relation to the bilinear form $(,)_{\mathfrak{h}}$. Then, the restriction of the bijection $\psi: \mathfrak{h} \longrightarrow \mathfrak{h}^{*}$ to $\mathfrak{h}_{ \pm}$provides the identification $\psi: \mathfrak{h}_{ \pm} \longrightarrow \mathfrak{h}_{\mp}^{*}$, and the restriction of the cocycle to the factors $G_{ \pm}$is then the map $C_{\mathrm{k}}: G_{ \pm} \rightarrow \mathfrak{h}_{\mp}^{*}$. Moreover, the bilinear form $(,)_{\mathfrak{h}}$ and the 2 cocycle $c_{\mathrm{k}}: \mathfrak{g} \times \mathfrak{g} \longrightarrow \mathbb{R}$ restricted to $\mathfrak{g}_{ \pm}$vanish, falling in the situation of Remark II of Sec. II B.

Thus, the Dirac bracket on the fiber $\mathcal{N}\left(g_{-}, \eta_{-}\right)$coincides with (4). This is the framework for the developments in the second part of this work.

\section{The left action of $G$ on $G \times \mathfrak{g}^{*}$ and its restriction to $\mathcal{N}\left(g_{-}, \eta_{-}\right)$}

This section is devoted to studying the left action of $G$ on $G \times \mathfrak{g}^{*}$ and how it restricts to the fibers $\mathcal{N}\left(g_{-}, \eta_{-}\right)$, analyzing the momentum maps borrowed from the phase space $G \times \mathfrak{g}^{*}$ equipped with the canonical symplectic structure.

The selected cocycle explicitly breaks the left action symmetry then it is no longer an endomorphism on $\left(T^{*} G, \omega_{c}\right)$. However, since the cocycle contribution seems to disappear from the Dirac brackets, we wonder whether that symmetry would be restored on some fibers $\mathcal{N}\left(g_{-}, \eta_{-}\right)$. If it were the case, the infinitesimal generator $X_{T^{*} G}$ would be related to the momentum functions associated with the canonical symplectic form, so we consider the momentum map associated with the left translation on $\left(T^{*} G, \omega_{\circ}\right)$, namely, $J_{B}^{L}: T^{*} G \longrightarrow \mathfrak{g}^{*}$ defined as

$$
J_{B}^{L}(g, \eta)=\operatorname{Ad}_{g^{-1}}^{G *} \eta
$$

It is worth stressing that it is not a momentum map for $\left(T^{*} G, \omega_{c}\right)$. Despite this fact, we shall consider the associated momentum function $j_{X}^{L}$, namely,

$$
j_{X}^{L}(g, \eta)=\left\langle\operatorname{Ad}_{g^{-1}}^{G *} \eta, X\right\rangle=\left\langle\eta, \operatorname{Ad}_{g^{-1}}^{G} X\right\rangle
$$

satisfying $l_{X_{T^{*}}} \omega_{\circ}=d j_{X}^{L}$, and construct the associated Hamiltonian vector fields. In doing so, we need the differential of $j_{X}^{L}$,

$$
d j_{X}^{L}=\left(g^{-1} \operatorname{ad}_{A d_{g^{-1}} X}^{\mathfrak{g} *} \eta, \operatorname{Ad}_{g^{-1}}^{G} X\right) .
$$

So, for an arbitrary function $\mathcal{F}$ on $G \times \mathfrak{g}^{*}$, its Lie derivative along the Hamiltonian vector field of $j_{X}^{L}$ projected on the tangent space to $\mathcal{N}\left(g_{-}, \eta_{-}\right)$is given by the Dirac bracket

$$
\begin{aligned}
\left\{\mathcal{F}, j_{X}^{L}\right\}_{c}^{D}(g, \eta)= & \left\langle g \mathbf{d} \mathcal{F}, \operatorname{Ad}_{g_{-}^{-1}}^{G} \Pi_{\mathfrak{g}_{+}} \operatorname{Ad}_{g_{+}^{-1}}^{G} X\right\rangle \\
& -\left\langle\operatorname{ad}_{A d_{g^{-1}} X}^{\mathfrak{g} *} \eta, \operatorname{Ad}_{g_{-}^{-1}}^{G} \Pi_{\mathfrak{g}_{+}} \operatorname{Ad}_{g_{-}}^{G} \delta \mathcal{F}\right\rangle \\
& -\left\langle\eta,\left[\operatorname{Ad}_{g_{-}^{-1}}^{G} \Pi_{\mathfrak{g}_{+}} \operatorname{Ad}_{g_{-}}^{G} \delta \mathcal{F}, \operatorname{Ad}_{g_{-}^{-1}}^{G} \Pi_{\mathfrak{g}_{+}} \operatorname{Ad}_{g_{+}^{-1}}^{G} X\right]\right\rangle
\end{aligned}
$$


so, the Hamiltonian vector field is

$$
V_{j_{X}^{L}}(g, \eta)=\left(g \operatorname{Ad}_{g_{-}^{-1}}^{G} \Pi_{\mathfrak{g}_{+}} \operatorname{Ad}_{g_{+}^{-1}}^{G} X,-\operatorname{Ad}_{g_{-}}^{G *} \Pi_{\mathfrak{g}_{+}^{*}} \operatorname{Ad}_{g_{-}^{-1}}^{G *} a d_{\operatorname{Ad}_{g_{-}}^{G} \Pi_{\mathfrak{g}_{-}} \operatorname{Ad}_{g_{+}^{-1}}^{G} X}^{\mathfrak{g} *} \eta\right) .
$$

For the fiber on $(e, 0)$ and $X=X_{+} \in \mathfrak{g}_{+}$we get

$$
V_{j_{X_{+}}^{L}}(g, \eta)=\left(X_{+} g_{+}, 0\right)
$$

which is just the infinitesimal generator of the left action of $G_{+}$on $T^{*} G_{+}$.

A test to see whether the symmetry is restored on $\mathcal{N}\left(g_{-}, \eta_{-}\right)$is to calculate the Dirac bracket of two momentum functions. If the result is that the momentum function closes a Lie algebra under the Dirac bracket, then we have a Lie algebra morphism between the Lie algebra of the group and the Lie algebra of momentum functions, showing that the symmetry is symplectically realized on $\mathcal{N}\left(g_{-}, \eta_{-}\right)$.

Proposition: Let $\eta_{-}$be a character of $\mathfrak{g}_{-}$. Then, the map $\mathfrak{g} \longrightarrow C^{\infty}\left(\mathcal{N}\left(g_{-}, \eta_{-}\right)\right)$such that $X \longrightarrow j_{X}^{L}$ is a Lie algebra homomorphism in relation to the Dirac bracket $(4)$.in $\mathcal{N}\left(g_{-}, \eta_{-}\right)$.

Proof: The Dirac bracket between momentum functions is

$$
\begin{aligned}
\left\{j_{X}^{L}, j_{Y}^{L}\right\}_{c}^{D}(g, \eta)= & \left\langle\eta,\left[\operatorname{Ad}_{g^{-1}}^{G} X, \operatorname{Ad}_{g_{-}^{-1}}^{G} \Pi_{\mathfrak{g}_{+}} \operatorname{Ad}_{g_{+}^{-1}}^{G} Y\right]\right\rangle \\
& +\left\langle\eta,\left[\operatorname{Ad}_{g_{-}^{-1}}^{G} \Pi_{\mathfrak{g}_{+}} \operatorname{Ad}_{g_{+}^{-1}}^{G} X, \operatorname{Ad}_{g_{-}^{-1}}^{G} \Pi_{\mathfrak{g}_{-}} \operatorname{Ad}_{g_{+}^{-1}}^{G} Y\right]\right\rangle .
\end{aligned}
$$

Observe that if $\eta_{-}$is a character of $\mathfrak{g}_{-}$we can add a term

$$
0=\left\langle\eta,\left[\operatorname{Ad}_{g_{-}^{-1}}^{G} \Pi_{\mathfrak{g}_{-}} \operatorname{Ad}_{g_{+}^{-1}}^{G} X, \operatorname{Ad}_{g_{-}^{-1}}^{G} \Pi_{\mathfrak{g}_{-}} \operatorname{Ad}_{g_{+}^{-1}}^{G} Y\right]\right\rangle,
$$

so $\left\{j_{X}^{L}, j_{Y}^{L}\right\}_{c}^{D}$ turns in to

$$
\left\{j_{X}^{L}, j_{Y}^{L}\right\}_{c}^{D}(g, \eta)=\left\langle\eta,\left[\operatorname{Ad}_{g^{-1}}^{G} X, \operatorname{Ad}_{g^{-1}}^{G} Y\right]\right\rangle
$$

which is equivalent to

$$
\left\{j_{X_{+}}^{L}, j_{Y}^{L}\right\}_{c}^{D}(g, \eta)=j_{[X, Y]}^{L}(g, \eta)
$$

indicating that the left invariance under the action of $G_{+}$is restored on $\mathcal{N}\left(g_{-}, \eta_{-}\right)$when $\eta_{-}$is a character of $\mathfrak{g}_{-}$.

\section{The action of the centrally extended group $G^{\wedge}$}

Now, we consider the action of the centrally extended loop group $G^{\wedge}$ that is more frequently involved in the Hamiltonian framework of loop groups with extended symplectic form concerning WZNW models, rather than the simple action of the group itself. So, it seems natural to work out the symmetries generated in this case by repeating the developments of Sec. II D.

Let $\mathfrak{g}_{c}$, the centrally extended Lie algebra $\mathfrak{g}$ be defined by the cocycle $c: \mathfrak{g} \otimes \mathfrak{g} \rightarrow \mathbb{R}$, and $\mathfrak{g}_{c}^{*}$ its dual algebra. The adjoint and coadjoint actions of $G$ coincide with those of the centrally extended Lie group $G^{\wedge}$ since the action of the extension factor on $\mathfrak{g}_{c}$ is trivial. Then, because $\mathbb{R}$ acts trivially, the adjoint action is

$$
\operatorname{Ad}_{(g, b)}^{G \wedge}(X, a):=\operatorname{Ad}_{g}^{G}(X, a)
$$

Explicitly, we have the formulas

$$
\left\{\begin{array}{l}
\operatorname{Ad}_{g}^{G}(X, a):=\left(\operatorname{Ad}_{g}^{G} X, a+\left\langle C\left(g^{-1}\right), X\right\rangle\right) \\
\operatorname{Ad}_{g^{-1}}^{G *}(\xi, b):=\left(\operatorname{Ad}_{g^{-1}}^{G *} \xi+b C(g), b\right) \\
\operatorname{ad}_{X}^{\mathfrak{g}}(Y, a):=([X, Y], c(X, Y)) .
\end{array}\right.
$$


Let us now introduce the action by left translations of $G^{\wedge}$ on $G \times \mathfrak{g}^{*}$ by defining the centrally extended momentum map

$$
J_{B}^{L \wedge}(g, \eta):=\operatorname{Ad}_{g^{-1}}^{G * \wedge}(\eta, 1)=\left(\operatorname{Ad}_{g^{-1}}^{G *} \eta+C(g), 1\right) .
$$

The momentum function $j_{X}^{L \wedge}$ associated with $J_{B}^{L \wedge}$, namely,

$$
j_{(X, a)}^{L \wedge}(g, \eta)=\left\langle\operatorname{Ad}_{g^{-1}}^{G * \wedge} \eta,(X, a)\right\rangle=j_{X}^{L}(g, \eta)+\langle C(g), X\rangle+a
$$

then

$$
d j_{(X, a)}^{L \wedge}=\left(g^{-1}\left(\operatorname{ad}_{A d_{g^{-1}} X}^{\mathfrak{g} *} \eta+\hat{c}\left(\operatorname{Ad}_{g^{-1}}^{G} X\right)\right), \operatorname{Ad}_{g^{-1}}^{G} X\right) .
$$

The centrally extended canonical symplectic form (1) gives rise to the Hamiltonian vector field

$$
V_{j_{(X, b)}^{L}}(g, \eta)=\left(X g, \operatorname{ad}_{A d_{g^{-1}}^{G} X}^{\mathfrak{g} *} C\left(g^{-1}\right)\right)
$$

and the Poisson bracket between momentum functions is

$$
\left\{j_{(X, a)}^{L \wedge}, j_{(Y, b)}^{L \wedge}\right\}_{c}(g, \eta)=\left\langle d j_{(X, a)}^{L \wedge}, V_{j_{(Y, b)}^{L}}\right\rangle_{(g, \eta)}
$$

and the explicit calculation gives

$$
\left\{j_{(X, a)}^{L \wedge}, j_{(Y, b)}^{L \wedge}\right\}_{c}(g, \eta)=j_{[(X, a),(Y, b)]}^{L \wedge}(g, \eta)+\langle C(g),[X, Y]\rangle
$$

which reflects the noninvariance of the symplectic form $\omega_{c}$.

Now, let us study the behavior of the Hamiltonian vector fields $V_{j_{(X, a)}^{L \wedge}} \equiv V_{(X, a)}^{\mathcal{N}}$ on the fibers $\mathcal{N}\left(g_{-}, \eta_{-}\right)$by calculating the Dirac brackets of these momentum functions. First, let us consider the Dirac bracket with an arbitrary function $\mathcal{F}$,

$$
\begin{aligned}
\left\{\mathcal{F}, j_{(X, a)}^{L \wedge}\right\}_{c}^{D}(g, \eta)= & \left\langle g \mathbf{d} \mathcal{F}, \operatorname{Ad}_{g_{-}^{-1}}^{G} \Pi_{\mathfrak{g}_{+}} \operatorname{Ad}_{g_{+}^{-1}}^{G} X\right\rangle \\
& -\left\langle\operatorname{ad}_{\operatorname{Ad}_{g^{-1}}^{G} X}^{\mathfrak{g} *} \eta+\hat{c}\left(\operatorname{Ad}_{g^{-1}}^{G} X\right), \operatorname{Ad}_{g_{-}^{-1}}^{G} \Pi_{\mathfrak{g}_{+}} \operatorname{Ad}_{g_{-}}^{G} \delta \mathcal{F}\right\rangle \\
& -\left\langle\eta,\left[\operatorname{Ad}_{g_{-}^{-1}}^{G} \Pi_{\mathfrak{g}_{+}} \operatorname{Ad}_{g_{-}}^{G} \delta \mathcal{F}, \operatorname{Ad}_{g_{-}^{-1}}^{G} \Pi_{\mathfrak{g}_{+}} \operatorname{Ad}_{g_{+}^{-1}}^{G} X\right]\right\rangle
\end{aligned}
$$

from where we get the Hamiltonian vector field

$$
\begin{aligned}
V_{(X, a)}^{\mathcal{N}}(g, \eta)= & \left(g \operatorname{Ad}_{g_{-}^{-1}}^{G} \Pi_{\mathfrak{g}_{+}} \operatorname{Ad}_{g_{+}^{-1}}^{G} X,\right. \\
& \left.-\operatorname{Ad}_{g_{-}}^{G *} \Pi_{\mathfrak{g}_{+}^{*}} \operatorname{Ad}_{g_{-}^{-1}}^{G *}\left(\operatorname{ad}_{\mathrm{Ad}_{g_{-}^{-1}}^{G} \Pi_{\mathfrak{g}_{-}} \operatorname{Ad}_{g_{+}^{-1}}^{G} X} \eta+\hat{c}\left(\operatorname{Ad}_{g^{-1}}^{G} X\right)\right)\right) .
\end{aligned}
$$

In an analogous way to the proposition at the end of Sec. II D, it is easy to see the following result.

Proposition: Let $\left(g_{-}, \eta_{-}\right) \in \operatorname{ker} C \times \operatorname{Char}\left(\mathfrak{g}_{-}\right)$, then the map $\mathfrak{g}^{\wedge} \longrightarrow C^{\infty}\left(\mathcal{N}\left(g_{-}, \eta_{-}\right)\right)$such that $(X, a) \longrightarrow j_{(X, a)}^{L}$ is a Lie algebra homomorphism in relation to the Dirac bracket (4) on $C^{\infty}\left(\mathcal{N}\left(g_{-}, \eta_{-}\right)\right)$, namely,

$$
\left\{j_{(X, a)}^{L \wedge}, j_{(Y, b)}^{L \wedge}\right\}_{c}^{D}(g, \eta)=j_{[(X, a),(Y, b)]}^{L \wedge}(g, \eta) .
$$

In terms of Hamiltonian vector fields it means that

$$
\left[V_{(X, a)}^{\mathcal{N}}, V_{(Y, b)}^{\mathcal{N}}\right]=-V_{[(X, a),(Y, b)]}^{\mathcal{N}}
$$

so, the linear map $\mathfrak{g}^{\wedge} \longrightarrow \mathfrak{X}\left(\mathcal{N}\left(g_{-}, \eta_{-}\right)\right) /(X, a) \longrightarrow V_{(X, a)}^{\mathcal{N}}$ is a Lie algebra antihomomorphism showing that it is the infinitesimal generator of a well-defined symplectic left action of $G^{\wedge}$ on $\mathcal{N}\left(g_{-}, \eta_{-}\right)$. 
As in Ref. 8, in the framework of $\left(T^{*} G, \omega_{\circ}\right)$, this infinitesimal action of $\mathfrak{g}$ on $\mathcal{N}\left(g_{-}, \eta_{-}\right)$gives rise to a finite action of $G^{\wedge}$ on $\mathcal{N}\left(g_{-}, \eta_{-}\right)$.

Proposition: The vector field $V_{(X, a)}^{\mathcal{N}} \in \mathfrak{X}\left(\mathcal{N}\left(g_{-}, \eta_{-}\right)\right)$, for $X \in \mathfrak{g}$ where $\eta_{-}$is a character of $\mathfrak{g}_{-}$, is the infinitesimal generator associated with the action $\mathrm{d}: G^{\wedge} \times \mathcal{N}\left(g_{-}, \eta_{-}\right) \rightarrow \mathcal{N}\left(g_{-}, \eta_{-}\right)$ = defined as

$$
\begin{aligned}
& \mathrm{d}((h, a),(g, \eta)) \\
= & \left(g \operatorname{Ad}_{g_{-}^{-1}}^{G} \Pi_{\mathfrak{g}_{+}}\left(g_{+}^{-1} h g_{+}\right),\right. \\
& \left.\operatorname{Ad}_{g_{-}}^{G *} \Pi_{\mathfrak{g}_{+}^{*}}\left(\operatorname{Ad}_{\Pi_{G_{-}}^{G *}\left(g_{+}^{-1} h g_{+}\right)} \operatorname{Ad}_{g_{-}}^{G *} \eta+C\left(\left(\Pi_{G_{-}}\left(g_{+}^{-1} h g_{+}\right)\right)^{-1}\right)\right)\right),
\end{aligned}
$$

$\forall(g, \eta)=\left(g_{+} g_{-}, \eta_{+}+\eta_{-}\right) \in \mathcal{N}\left(g_{-}, \eta_{-}\right)$.

Proof: It follows by straightforward calculation of the differential of this map.

Remark: Observe that for $g_{-}=e$ and $\eta_{-}=0$ it turns into

$$
\begin{aligned}
& \mathrm{d}((h, a),(g, \eta)) \\
= & \left(g_{+} \Pi_{\mathfrak{g}_{+}}\left(g_{+}^{-1} h g_{+}\right),\right. \\
& \left.\Pi_{\mathfrak{g}_{+}^{*}}\left(\operatorname{Ad}_{\Pi_{G_{-}}^{G *}\left(g_{+}^{-1} h g_{+}\right)} \eta_{+}+C\left(\left(\Pi_{G_{-}}\left(g_{+}^{-1} h g_{+}\right)\right)^{-1}\right)\right)\right) .
\end{aligned}
$$

This action was introduced in Refs. 6 and 7 as the fundamental ingredient underlying the Hamiltonian Poisson Lie T-duality scheme.

So far we have shown that, although there is no left translation symmetry on the phase space $\left(T^{*} G, \omega_{c}\right)$, it is restored on some particular fibers $\mathcal{N}\left(g_{-}, \eta_{-}\right)$turning them into potentially interesting phase space for symmetric systems under the projection on $\mathcal{N}\left(g_{-}, \eta_{-}\right)$of the left action of $G^{\wedge}$ on $T^{*} G$.

\section{E. The Hamilton equations and collective dynamics}

In order to study dynamical systems with the kind of phase space described above, we explicitly write both Hamilton equations in the whole space $T^{*} G$ and those on the constrained submanifolds $\mathcal{N}\left(g_{-}, \eta_{-}\right)$produced by the Dirac brackets, for a generic Hamilton function $\mathcal{H}$ on $T^{*} G$.

The Hamilton equations on $T^{*} G$ are determined by the Hamiltonian vector field associated with a Hamilton function $\mathcal{H}$ through the symplectic form (1) that was given in (2). So, defining $d \mathcal{H}=(\mathbf{d} \mathcal{H}, \delta \mathcal{H}) \in T_{(g, \eta)}^{*}\left(G \times \mathfrak{g}^{*}\right)$, these Hamilton equations are

$$
\left\{\begin{array}{l}
g^{-1} \dot{g}=\delta \mathcal{H} \\
\dot{\eta}=\operatorname{ad}_{\delta \mathcal{H}}^{\mathfrak{g} *} \eta-g \mathbf{d} \mathcal{H}+\operatorname{Ad}_{g}^{G *} \hat{c}\left(\operatorname{Ad}_{g}^{G} \delta \mathcal{H}\right) .
\end{array}\right.
$$

From the Hamiltonian vector field (5), which is tangent to $\mathcal{N}\left(g_{-}, \eta_{-}\right)$, we get the Hamilton equations on this phase space:

$$
\left\{\begin{array}{l}
g^{-1} \dot{g}=\operatorname{Ad}_{g_{-}^{-1}}^{G} \Pi_{\mathfrak{g}_{+}} \operatorname{Ad}_{g_{-}}^{G} \delta \mathcal{H} \\
\dot{\eta}=\operatorname{Ad}_{g_{-}}^{G *} \Pi_{\mathfrak{g}_{+}^{*}} \operatorname{Ad}_{g_{-}^{-1}}^{G *}\left(\operatorname{ad}_{\operatorname{Ad}_{g_{-}^{-1}}^{G} \Pi_{\mathfrak{g}_{+}} \operatorname{Ad}_{g_{-}}^{G} \delta \mathcal{H}}^{\mathfrak{g}} \eta-g \mathbf{d} \mathcal{H}\right) .
\end{array}\right.
$$


In terms of the components in $G_{ \pm}$and $\mathfrak{g}_{ \pm}^{*}$ they means

$$
\left\{\begin{array}{l}
g_{+}^{-1} \dot{g}_{+}=\Pi_{\mathfrak{g}_{+}} \operatorname{Ad}_{g_{-}}^{G} \delta \mathcal{H} \\
\dot{g}_{-}=0 \\
\dot{\eta}_{+}=\operatorname{Ad}_{g_{-}}^{G *} \Pi_{\mathfrak{g}_{+}^{*}} \operatorname{Ad}_{g_{-}^{-1}}^{G *}\left(\operatorname{ad}_{\operatorname{Ad}_{g_{-}}^{G} \Pi_{\mathfrak{g}_{+}} \operatorname{Ad}_{g_{-}}^{G} \delta \mathcal{H}} \eta-g \mathbf{d} \mathcal{H}\right) \\
\dot{\eta}_{-}=0
\end{array}\right.
$$

We shall be concerned with a dynamical system ruled by a Hamilton function of the type

$$
\mathcal{H}=\mathrm{h} \circ J_{B}^{L \wedge} / \mathrm{h}: \mathfrak{g}^{\wedge *} \longrightarrow \mathbb{R}
$$

with $J_{B}^{L \wedge}$ being the momentum map associated with the centrally extended left translation on ( $T^{*} G, \omega_{\circ}$ ) given in Eq. (6). Although it is apparently of a collective type, actually that is not the case because $J_{B}^{L \wedge}$ fails to be a momentum map for $\left(T^{*} G, \omega_{c}\right)$, which lacks left invariance symmetry.

However, it is a truly collective system on $\mathcal{N}\left(g_{-}, \eta_{-}\right)$, for $\left(g_{-}, \eta_{-}\right) \in \operatorname{ker} C \times \operatorname{Char}\left(\mathfrak{g}_{-}\right)$, where the centrally extended left symmetry is restored. In these fibers, the dynamics acquires interesting properties that we briefly describe now. ${ }^{13}$ The derivative of some function $\mathcal{F}$ along the flux of the Hamiltonian vector field (5) is

$$
\dot{\mathcal{F}}=\mathbf{L}_{V_{\mathcal{H}}} \mathcal{F}=\{\mathcal{F}, \mathcal{H}\}_{c}^{D}
$$

Let us denote by $\varphi_{(g, \eta)}: G^{\wedge} \longrightarrow \mathcal{N}\left(g_{-}, \eta_{-}\right)$, the orbit map associated with the action (8) such that

$$
\varphi_{(g, \eta)}(h, a)=\mathrm{d}((h, a),(g, \eta))
$$

then, its differential at the neutral element of $G^{\wedge}$ is the map $\varphi_{(g, \eta) *}: \mathfrak{g}^{\wedge} \longrightarrow T_{(g, \eta)} \mathcal{N}\left(g_{-}, \eta_{-}\right)$gives rise to the infinitesimal generator $V_{(X, a)}^{\mathcal{N}}$ on $\mathcal{N}\left(g_{-}, \eta_{-}\right)$defined in (7), then

$$
\varphi_{(g, \eta) *}(X, a):=V_{(X, a)}^{\mathcal{N}}(g, \eta) .
$$

The Hamiltonian vector field associated with $\mathcal{H}=\mathrm{h} \circ J_{B}^{L \wedge}$ is then

$$
V_{\mathcal{H}}(g, \eta)=\varphi_{(g, \eta) *} \mathcal{L}_{\mathrm{h}}\left(J_{B}^{L \wedge}(g, \eta)\right),
$$

where $\mathcal{L}_{\mathrm{h}}: \mathfrak{g}^{\wedge *} \longrightarrow \mathfrak{g}^{\wedge}$ is defined as

$$
\left\langle\xi, \mathcal{L}_{\mathrm{h}}(\eta, b)\right\rangle:=\left.\frac{d}{d t} \mathrm{~h}(\eta+t \xi, b)\right|_{t=0}
$$

meaning that $V_{\mathcal{H}}(g, \eta)$ coincides with the infinitesimal generator associated with $\mathcal{L}_{\mathrm{h}}\left(J_{B}^{L \wedge}(g, \eta)\right) \in$ $\mathfrak{g}^{\wedge}$. So, (10) is

$$
\dot{\mathcal{F}}=\left\{\mathcal{F}, j_{\mathcal{L}_{\mathrm{h} \circ J_{B}^{L \wedge}}^{L \wedge}}\right\}_{c}^{D}
$$

In this way, at least locally, the integral curves of the Hamiltonian vector field are orbits $\mathrm{d}((h(t), a),(g, \eta))$ of a curve $\gamma: \mathbb{R} \longrightarrow G / t \stackrel{\gamma}{\longmapsto} h(t)$. By the way, this curve is the same that solves the problem

$$
\frac{d}{d t}(\eta, b)=\operatorname{ad}_{\mathcal{L}_{\mathrm{h}}(\eta, b)}^{\mathfrak{g} \wedge *}(\eta, b)
$$

which is equivalent to this one

$$
\left\{\begin{array}{l}
(\eta, b)(t)=\operatorname{Ad}_{(h(t), a)}^{G \wedge *}(\eta, b)\left(t_{\mathrm{o}}\right) \\
(h(t), a)^{-1} \frac{d}{d t}(h(t), a)=\mathcal{L}_{\mathrm{h}}(\eta, b) .
\end{array}\right.
$$


Thus, the dynamical system on $\mathcal{N}\left(g_{-}, \eta_{-}\right)$is replicated on the same coadjoint orbit in $\mathfrak{g}^{\wedge *}$, and the Ad-equivariance for the momentum maps allows us to translate orbits in $\mathfrak{g}^{\wedge *}$ into orbits in $\mathcal{N}\left(g_{-}, \eta_{-}\right)$.

\section{PART II}

\section{FROM A WZNW TYPE MODEL TO THE POISSON-LIE $\sigma$-MODEL}

As the main application of the results of the first part, we study a Hamiltonian system that turns into a collective type on the phase subspaces $\mathcal{N}\left(g_{-}, \eta_{-}\right)$, for $\left(g_{-}, \eta_{-}\right) \in \operatorname{ker} C \times \operatorname{Char}\left(\mathfrak{g}_{-}\right)$. By deriving the Hamilton equations, we retrieve the Lagrange version of the model, showing that it corresponds to a generalization of the so-called Poisson-Lie $\sigma$-model, namely, a Poisson sigma modelwith target in a Poisson-Lie group. ${ }^{2,5,12}$

\section{A. A collective Hamilton function on $\mathcal{N}\left(g_{-}, \eta_{-}\right)$}

Motivated by the previous observation, we now propose studying a Hamiltonian system ruled by the Hamilton function

$$
\mathcal{H}(g, \eta)=\frac{1}{2}\left(\psi\left(J_{B}^{L \wedge}(g, \eta)\right), \mathcal{E} \psi\left(J_{B}^{L \wedge}(g, \eta)\right)\right)_{\mathfrak{g}^{\wedge}},
$$

where $J_{B}^{L \wedge}(g, \eta)$ is the centrally extended momentum map associated with left translations on the phase space $\left(T^{*} G, \omega_{\circ}\right)$, introduced in Eq. (6). The linear operator $\mathcal{E}: \mathfrak{g} \rightarrow \mathfrak{g}$ is idempotent $\mathcal{E}^{2}=I d$, and breaks the Ad-invariance of the bilinear form. For further issues we introduce the operator $\mathcal{E}_{g}: \mathfrak{g} \rightarrow \mathfrak{g}$.

Definition: Let the operator $\mathcal{E}_{g}: \mathfrak{g} \rightarrow \mathfrak{g}$ be defined as

$$
\mathcal{E}_{g}=\operatorname{Ad}_{g^{-1}}^{G} \mathcal{E}_{\mathrm{Ad}_{g}^{G}}^{G}=\left(\begin{array}{cc}
-\mathcal{G}_{g}^{-1} \mathcal{B}_{g} & \mathcal{G}_{g}^{-1} \\
\mathcal{G}_{g}-\mathcal{B}_{g} \mathcal{G}_{g}^{-1} \mathcal{B}_{g} & \mathcal{B}_{g} \mathcal{G}_{g}^{-1}
\end{array}\right),
$$

where

$$
\left\{\begin{array}{l}
\mathcal{G}_{g}=\left(\Pi_{\mathfrak{g}_{+}} \mathcal{E}_{g} \Pi_{\mathfrak{g}_{-}}\right)^{-1}: \mathfrak{g}_{+} \longrightarrow \mathfrak{g}_{-} /\left\langle\mathcal{G}_{g} X, Y\right\rangle=\left\langle X, \mathcal{G}_{g} Y\right\rangle \\
\mathcal{B}_{g}=-\mathcal{G}_{g} \circ \Pi_{\mathfrak{g}_{+}} \mathcal{E}_{g} \Pi_{\mathfrak{g}_{+}}: \mathfrak{g}_{+} \longrightarrow \mathfrak{g}_{-} /\left\langle\mathcal{B}_{g} X, Y\right\rangle=-\left\langle X, \mathcal{B}_{g} Y\right\rangle
\end{array}\right.
$$

which makes $\mathcal{E}_{g}$ symmetric

$$
\left((Y, \xi), \mathcal{E}_{g}(X, \eta)\right)_{L \mathfrak{d}}=\left(\mathcal{E}_{g}(Y, \xi),(X, \eta)\right)_{L \mathfrak{d}}
$$

and idempotent

$$
\mathcal{E}_{g} \circ \mathcal{E}_{g}=I d_{L \mathfrak{O}} .
$$

It gives rise to a decomposition of $\mathfrak{g}$ in two eigenvalue subspaces

$$
\mathcal{E}^{ \pm}(g)=\left\{X \in \mathfrak{g} / \mathcal{E}_{g} X= \pm X\right\}
$$

such that $\mathfrak{g}=\mathcal{E}^{+} \oplus \mathcal{E}^{-}$. Therefore, if $X$ is in $\mathcal{E}^{ \pm}(g)$ it can be written as

$$
X=\left(X_{+},\left(\mathcal{B}_{g} \pm \mathcal{G}_{g}\right) X_{+}\right)
$$

or, alternatively, as

$$
X=\left(\left(\mathcal{B}_{g} \pm \mathcal{G}_{g}\right)^{-1} X_{-}, X_{-}\right) .
$$

Observe that if $X \in \mathcal{E}^{ \pm}(g)$, then $\operatorname{Ad}_{g_{+}}^{G} X \in \mathcal{E}^{ \pm}(e)$. 
Coming back to the Hamilton function on $G \times \mathfrak{g}^{*}$, we write

$$
\mathcal{H}(g, \eta)=\frac{1}{2}\left(\bar{\psi}\left(\operatorname{Ad}_{g^{-1}}^{G *} \eta+C(g)\right), \mathcal{E} \bar{\psi}\left(\operatorname{Ad}_{g^{-1}}^{G *} \eta+C(g)\right)\right)_{\mathfrak{g}}
$$

and its differential $d \mathcal{H}=(\mathbf{d} \mathcal{H}, \delta \mathcal{H})$ is

$$
\left\{\begin{aligned}
\mathbf{d} \mathcal{H} & =g^{-1} \psi\left[\bar{\psi}\left(\eta-C\left(g^{-1}\right)\right), \mathcal{E}_{g} \bar{\psi}\left(\eta-C\left(g^{-1}\right)\right)\right] \\
& +g^{-1} \operatorname{Ad}_{g}^{G *} \hat{c}\left(\mathcal{E} \operatorname{Ad}_{g}^{G} \bar{\psi}\left(\eta-C\left(g^{-1}\right)\right)\right) \\
\delta \mathcal{H} & =\mathcal{E}_{g} \bar{\psi}\left(\eta-C\left(g^{-1}\right)\right) .
\end{aligned}\right.
$$

The Hamilton equations in $G \times \mathfrak{g}^{*}$ are obtained from the Hamiltonian vector field (2) giving

$$
\left\{\begin{array}{l}
g^{-1} \dot{g}=\mathcal{E}_{g} \bar{\psi}\left(\eta-C\left(g^{-1}\right)\right) \\
\dot{\eta}=\operatorname{ad}_{\mathcal{E}_{g} \bar{\psi}\left(\eta-C\left(g^{-1}\right)\right)}^{\mathfrak{g} *} C\left(g^{-1}\right) .
\end{array}\right.
$$

It is worth noting remark here that, in retrieving the Lagrange equation, we get the second order equation

$$
\frac{\partial}{\partial t}\left(\mathcal{E}_{g}\left(g^{-1} \dot{g}\right)+\bar{\psi}\left(C\left(g^{-1}\right)\right)\right)=\left[g^{-1} \dot{g}, \bar{\psi}\left(C\left(g^{-1}\right)\right)\right],
$$

where the Lie algebra bracket on the rhs is a manifestation of the topological WZ term.

\section{The Hamilton equation in $\mathcal{N}\left(g_{-}, \eta_{-}\right)$}

The Hamilton equation in $\mathcal{N}\left(g_{-}, \eta_{-}\right)$was given in (9). Since we shall be concerned with loop groups, from now on we assume that the restrictions of the cocycle $C$ to the factors $G_{ \pm}$have their images contained in $\mathfrak{g}_{\mp}^{*}$, respectively, so $C: G_{ \pm} \rightarrow \mathfrak{g}_{\mp}^{*}$.

Then, replacing $\mathbf{d} \mathcal{H}, \delta \mathcal{H}$ in those Hamilton equation by the ones given in (12) we get the Hamilton equations for the Hamilton function (11). Before writing them in terms of the components $G_{ \pm} \times \mathfrak{g}_{ \pm}^{*}$, we verify that they are indeed generated by an infinitesimal generator of the action (8). In fact, after replacing the differential (12) in Eqs. (9), we can handle them to the form

$$
\left\{\begin{array}{c}
g^{-1} \dot{g}=\operatorname{Ad}_{g_{-}^{-1}}^{G} \Pi_{\mathfrak{g}_{+}} \operatorname{Ad}_{g_{+}^{-1}}^{G}\left(\operatorname{Ad}_{g}^{G} \mathcal{E}_{g} \bar{\psi}\left(\eta-C\left(g^{-1}\right)\right)\right) \\
\dot{\eta}=-\operatorname{Ad}_{g_{-}}^{G *} \Pi_{\mathfrak{g}_{+}^{*}} \operatorname{Ad}_{g_{-}^{-1}}^{G *}\left(\operatorname{ad}_{\left.\mathrm{Ad}_{g_{-}^{-1}}^{G} \Pi_{\mathfrak{g}_{-}} \operatorname{Ad}_{g_{+}^{-1}}^{G}\left(\operatorname{Ad}_{g}^{G} \mathcal{E}_{g} \bar{\psi}\left(\eta-C\left(g^{-1}\right)\right)\right)^{\eta}\right)}^{\eta} \operatorname{Ad}_{g_{-} *}^{G *} \Pi_{\mathfrak{g}_{+}^{*}} \operatorname{Ad}_{g_{-}^{-1}}^{G *}\left(\hat{c}\left(\operatorname{Ad}_{g^{-1}}^{G}\left(\operatorname{Ad}_{g}^{G} \mathcal{E}_{g} \bar{\psi}\left(\eta-C\left(g^{-1}\right)\right)\right)\right)\right)\right.
\end{array}\right.
$$

verifying that

$$
(\dot{g}, \dot{\eta})=V_{\left(\operatorname{Ad}_{g}^{G} \mathcal{E}_{g} \bar{\psi}\left(\eta-C\left(g^{-1}\right)\right), a\right)}^{\mathcal{N}}(g, \eta)
$$

for $V_{(X, a)}^{\mathcal{N}}$ defined in (7), as we expected from the collective character of the dynamics in $\mathcal{N}\left(g_{-}, \eta_{-}\right)$.

Now, let us work out the dynamics in $\mathcal{N}\left(g_{-}, \eta_{-}\right)$in terms of its components $G_{ \pm} \times \mathfrak{g}_{ \pm}^{*}$ by writing $g=g_{+} g_{-}$and $\eta=\eta_{+}+\eta_{-}$. Of course, $\left(g_{-}, \eta_{-}\right)$remains frozen, while $\left(g_{+}, \eta_{+}\right)$evolves in $\mathcal{N}\left(g_{-}, \eta_{-}\right)$ruled by the equations

$$
\left\{\begin{array}{l}
g_{+}^{-1} \dot{g}_{+}=\Pi_{\mathfrak{g}_{+}} \operatorname{Ad}_{g_{+}^{-1}}^{G} \mathcal{E} \operatorname{Ad}_{g}^{G} \bar{\psi}\left(\eta-C\left(g^{-1}\right)\right) \\
\dot{\eta}=-\operatorname{Ad}_{g_{-}}^{G *} \Pi_{\mathfrak{g}_{+}^{*}} \psi\left[\operatorname{Ad}_{g_{-}}^{G} \bar{\psi}\left(\eta-C\left(g^{-1}\right)\right), \Pi_{\mathfrak{g}_{-}} \operatorname{Ad}_{g_{-}}^{G} \mathcal{E}_{g} \bar{\psi}\left(\eta-C\left(g^{-1}\right)\right)\right] \\
\quad+\operatorname{Ad}_{g_{-}}^{G *} \Pi_{\mathfrak{g}_{+}^{*}} \psi\left[\operatorname{Ad}_{g_{-}}^{G} \bar{\psi}\left(C\left(g^{-1}\right)\right), \Pi_{\mathfrak{g}_{+}} \operatorname{Ad}_{g_{-}}^{G} \mathcal{E}_{g} \bar{\psi}\left(\eta-C\left(g^{-1}\right)\right)\right] \\
\quad-\operatorname{Ad}_{g_{-}}^{G *} \Pi_{\mathfrak{g}_{+}^{*}} \operatorname{Ad}_{g_{+}}^{G *} \hat{c}\left(\mathcal{E} \operatorname{Ad}_{g}^{G} \bar{\psi}\left(\eta-C\left(g^{-1}\right)\right)\right) .
\end{array}\right.
$$

Here we observe that, by taking $\left(g_{-}, \eta_{-}\right) \in \operatorname{ker} C \times \operatorname{Char}\left(\mathfrak{g}_{-}\right)$it follows that

$$
C\left(g^{-1}\right)=C\left(g_{-}^{-1} g_{+}^{-1}\right)=\operatorname{Ad}_{g_{-}}^{G *} C\left(g_{+}^{-1}\right) .
$$


Then, for $\eta_{ \pm} \in \mathfrak{g}_{ \pm}^{*}$ and $g_{\mp} \in G_{\mp}, \operatorname{Ad}_{g_{\mp} *}^{G *} \eta_{ \pm} \in \mathfrak{g}_{ \pm}^{*}$, which also means that $\operatorname{ad}_{X_{\mp}}^{\mathfrak{g} *} \eta_{ \pm}=\Pi_{\mathfrak{g}_{+}^{*}} \operatorname{ad}_{X_{\mp}}^{\mathfrak{g} *} \eta_{ \pm}$. Again, using these facts and the properties of the different cocycles, we arrive at

$$
\left\{\begin{array}{l}
g_{+}^{-1} \dot{g}_{+}=\Pi_{\mathfrak{g}_{+}} \mathcal{E}_{g_{+}} \bar{\psi}\left(\operatorname{Ad}_{g_{-}^{-1}}^{G *} \eta-C\left(g_{+}^{-1}\right)\right) \\
\operatorname{Ad}_{g_{-}^{-1}}^{G *} \dot{\eta}=-\Pi_{\mathfrak{g}_{+}^{*}} \psi\left[\bar{\psi}\left(\operatorname{Ad}_{g_{-}^{-1}}^{G *} \eta\right), \Pi_{\mathfrak{g}_{-}} \mathcal{E}_{g_{+}} \bar{\psi}\left(\operatorname{Ad}_{g_{-}^{-1}}^{G *} \eta-C\left(g_{+}^{-1}\right)\right)\right] \\
-\Pi_{\mathfrak{g}_{+}^{*}} \hat{c}\left(\Pi_{\mathfrak{g}_{-}} \mathcal{E}_{g_{+}} \bar{\psi}\left(\operatorname{Ad}_{g_{-}^{-1}}^{G *} \eta-C\left(g_{+}^{-1}\right)\right)\right) .
\end{array}\right.
$$

\section{B. The Lagrange equation}

We now proceed to retrieve the Lagrange equation on $\mathcal{N}\left(g_{-}, \eta_{-}\right)$by replacing the first Hamilton equation into the second one. Therefore, since $\eta_{-}$is a character of $\mathfrak{g}_{-}$,

$$
\Pi_{\mathfrak{g}_{-}^{*}} \operatorname{Ad}_{g_{-}^{-1}}^{G *} \eta_{-}=A d_{g_{-}^{-1}}^{*} \eta_{-}=\eta_{-}
$$

the first Hamilton equation becomes

$$
g_{+}^{-1} \dot{g}_{+}=\mathcal{G}_{g_{+}}^{-1} \bar{\psi}\left(\operatorname{Ad}_{g_{-}^{-1}}^{G *} \eta_{+}\right)+\mathcal{G}_{g_{+}}^{-1} \bar{\psi}\left(\Pi_{\mathfrak{g}_{+}^{*}} \operatorname{Ad}_{g_{-}^{-1}}^{G *} \eta_{-}\right)+\mathcal{G}_{g_{+}}^{-1} \mathcal{B}_{g_{+}} \bar{\psi}\left(C\left(g_{+}^{-1}\right)-\eta_{-}\right)
$$

then

$$
\bar{\psi}\left(\operatorname{Ad}_{g_{-}^{-1}}^{G *} \eta_{+}\right)=\mathcal{G}_{g_{+}} g_{+}^{-1} \dot{g}_{+}-\mathcal{B}_{g_{+}} \bar{\psi}\left(C\left(g_{+}^{-1}\right)\right)+\left(\mathcal{B}_{g_{+}}-\Pi_{\mathfrak{g}_{-}} \operatorname{Ad}_{g_{-}}^{G}\right) \bar{\psi}\left(\eta_{-}\right) .
$$

The second Hamilton equation can be written as

$$
\begin{aligned}
& \bar{\psi}\left(A d_{g_{-}^{-1}}^{G *} \dot{\eta}\right) \\
= & -\Pi_{\mathfrak{g}_{-}}\left[\bar{\psi}\left(\operatorname{Ad}_{g_{-}^{-1}}^{G *} \eta\right),\left(\mathcal{G}_{g_{+}}-\mathcal{B}_{g_{+}}\left(\mathcal{G}_{g_{+}}\right)^{-1} \mathcal{B}_{g_{+}}\right) \Pi_{\mathfrak{g}_{+}} \bar{\psi}\left(\operatorname{Ad}_{g_{-}^{-1}}^{G *} \eta-C\left(g_{+}^{-1}\right)\right)\right] \\
& -\Pi_{\mathfrak{g}_{-}}\left[\bar{\psi}\left(\operatorname{Ad}_{g_{-}^{-1}}^{G *} \eta\right), \mathcal{B}_{g_{+}}\left(\mathcal{G}_{g_{+}}\right)^{-1} \Pi_{\mathfrak{g}_{-}} \bar{\psi}\left(\operatorname{Ad}_{g_{-}^{-1}}^{G *} \eta-C\left(g_{+}^{-1}\right)\right)\right] \\
& -\bar{\psi}\left(\hat{c}\left(\left(\mathcal{G}_{g_{+}}-\mathcal{B}_{g_{+}}\left(\mathcal{G}_{g_{+}}\right)^{-1} \mathcal{B}_{g_{+}}\right) \Pi_{\mathfrak{g}_{+}} \bar{\psi}\left(\operatorname{Ad}_{g_{-}^{-1}}^{G *} \eta-C\left(g_{+}^{-1}\right)\right)\right)\right) \\
& -\bar{\psi}\left(\hat{c}\left(\mathcal{B}_{g_{+}}\left(\mathcal{G}_{g_{+}}\right)^{-1} \Pi_{\mathfrak{g}_{-}} \bar{\psi}\left(\operatorname{Ad}_{g_{-}^{-}}^{G *} \eta-C\left(g_{+}^{-1}\right)\right)\right)\right)
\end{aligned}
$$

and by substituting $\bar{\psi}\left(A d_{g_{-}^{-1}}^{G *} \eta_{+}\right)$as given in Eq. (13) we get

$$
\begin{aligned}
& \frac{d}{d t}\left(\mathcal{G}_{g_{+}} g_{+}^{-1} \dot{g}_{+}-\mathcal{B}_{g_{+}} \bar{\psi}\left(C\left(g_{+}^{-1}\right)\right)+\mathcal{B}_{g_{+}} \bar{\psi}\left(\eta_{-}\right)\right) \\
& =-\Pi_{\mathfrak{g}_{-}}\left[\mathcal{G}_{g_{+}} g_{+}^{-1} \dot{g}_{+}-\mathcal{B}_{g_{+}} \bar{\psi}\left(C\left(g_{+}^{-1}-\eta_{-}\right)\right), \mathcal{B}_{g_{+}} g_{+}^{-1} \dot{g}_{+}-\mathcal{G}_{g_{+}} \bar{\psi}\left(C\left(g_{+}^{-1}\right)-\eta_{-}\right)\right] \\
& -\Pi_{\mathfrak{g}_{-}}\left[\bar{\psi}\left(\eta_{-}\right), \mathcal{B}_{g_{+}} g_{+}^{-1} \dot{g}_{+}-\mathcal{G}_{g_{+}} \bar{\psi}\left(C\left(g_{+}^{-1}\right)-\eta_{-}\right)\right] \\
& -\bar{\psi}\left(\hat{c}\left(\mathcal{B}_{g_{+}} g_{+}^{-1} \dot{g}_{+}-\mathcal{G}_{g_{+}} \bar{\psi}\left(C\left(g_{+}^{-1}\right)-\eta_{-}\right)\right)\right) .
\end{aligned}
$$

On the fiber $\mathcal{N}(e, 0) \cong G_{+} \times \mathfrak{g}_{+}^{*}$ it reduces to

$$
\begin{aligned}
& \frac{d}{d t} \psi\left(\mathcal{G}_{g_{+}} g_{+}^{-1} \dot{g}_{+}-\mathcal{B}_{g_{+}} \bar{\psi}\left(C\left(g_{+}^{-1}\right)\right)\right)+\hat{c}\left(\mathcal{B}_{g_{+}} g_{+}^{-1} \dot{g}_{+}-\mathcal{G}_{g_{+}} \bar{\psi}\left(C\left(g_{+}^{-1}\right)\right)\right) \\
= & -\psi\left[\mathcal{G}_{g_{+}} g_{+}^{-1} \dot{g}_{+}-\mathcal{B}_{g_{+}} \bar{\psi}\left(C\left(g_{+}^{-1}\right)\right), \mathcal{B}_{g_{+}} g_{+}^{-1} \dot{g}_{+}-\mathcal{G}_{g_{+}} \bar{\psi}\left(C\left(g_{+}^{-1}\right)\right)\right] .
\end{aligned}
$$

\section{The Lagrangian function on $\mathcal{N}\left(g_{-}, \eta_{-}\right)$}

Now, we build up the Lagrangian version on the fibers $\mathcal{N}\left(g_{-}, \eta_{-}\right)$, which in turn are symplectic submanifolds equipped with the restriction of the symplectic form on $G \times \mathfrak{g}^{*}$. The Lagrangian 
function relates to the Hamilton function by

$$
d L=\Theta-d H
$$

provided there exists the 1 -form $\Theta$ satisfying

$$
\omega_{c} \mid \mathcal{N}\left(g_{-}, \eta_{-}\right)=-d \Theta .
$$

In the current case, it is supplied by the following proposition.

Proposition: The restriction of the symplectic form $\omega_{c}$ to $\mathcal{N}\left(g_{-}, \eta_{-}\right)$is an exact form such that

$$
\omega_{c} \mid \mathcal{N}\left(g_{-}, \eta_{-}\right)=-d \Theta
$$

with $\Theta$ being the restriction of the canonical 1-form of $G \times \mathfrak{g}^{*}$ to $\mathcal{N}\left(g_{-}, \eta_{-}\right)$,

$$
\left\langle\Theta,\left(g_{+}\left(\bar{\psi}\left(A d_{g_{-}^{-1}}^{*} \psi\left(X_{+}\right)\right)\right) g_{-}, \xi_{+}\right)\right\rangle_{(g, \eta)}:=\left\langle\eta, g_{+}\left(\bar{\psi}\left(A d_{g_{-}^{-1}}^{*} \psi\left(X_{+}\right)\right)\right) g_{-}\right\rangle .
$$

Proof: By the isotropic character of the subspaces $\mathfrak{g}_{ \pm}$under the cocycle $c$, the restriction of $\omega_{c}$ reduces to

$$
\begin{aligned}
& \left\langle\omega,\left(g_{+}\left(\bar{\psi}\left(A d_{g_{-}^{-1}}^{*} \psi\left(X_{+}\right)\right)\right) g_{-}, \xi_{+}\right) \otimes\left(g_{+}\left(\bar{\psi}\left(A d_{g_{-}^{-1}}^{*} \psi\left(Y_{+}\right)\right)\right) g_{-}, \lambda_{+}\right)\right\rangle_{(g, \eta)} \\
= & -\left\langle\xi_{+}, \operatorname{Ad}_{g_{-}^{-1}}^{G}\left(\bar{\psi}\left(A d_{g_{-}^{-1}}^{*} \psi\left(Y_{+}\right)\right)\right)\right\rangle+\left\langle\lambda_{+}, \operatorname{Ad}_{g_{-}^{-1}}^{G}\left(\bar{\psi}\left(A d_{g_{-}^{-1}}^{*} \psi\left(X_{+}\right)\right)\right)\right\rangle \\
& +\left\langle\eta,\left[\operatorname{Ad}_{g_{-}^{-1}}^{G}\left(\bar{\psi}\left(A d_{g_{-}^{-1}}^{*} \psi\left(X_{+}\right)\right)\right), \operatorname{Ad}_{g_{-}^{-1}}^{G}\left(\bar{\psi}\left(A d_{g_{-}^{-1}}^{*} \psi\left(Y_{+}\right)\right)\right)\right]\right\rangle .
\end{aligned}
$$

Let us define it as $\Theta \in T^{*} \mathcal{N}\left(g_{-}, \eta_{-}\right)$such that

$$
\left\langle\Theta,\left(g_{+}\left(\bar{\psi}\left(A d_{g_{-}^{-1}}^{*} \psi\left(X_{+}\right)\right)\right) g_{-}, \xi_{+}\right)\right\rangle_{(g, \eta)}:=\left\langle\eta, g_{+}\left(\bar{\psi}\left(A d_{g_{-}^{-1}}^{*} \psi\left(X_{+}\right)\right)\right) g_{-}\right\rangle
$$

then

$$
\begin{aligned}
& \left\langle d \Theta,\left(g_{+}\left(\bar{\psi}\left(A d_{g_{-}^{-1}}^{*} \psi\left(X_{+}\right)\right)\right) g_{-}, \xi_{+}\right) \otimes\left(g_{+}\left(\bar{\psi}\left(A d_{g_{-}^{-1}}^{*} \psi\left(Y_{+}\right)\right)\right) g_{-}, \lambda_{+}\right)\right\rangle_{(g, \eta)} \\
= & \left\langle\xi_{+}, g_{+}\left(\bar{\psi}\left(A d_{g_{-}^{-1}}^{*} \psi\left(Y_{+}\right)\right)\right) g_{-}\right\rangle-\left\langle\lambda_{+}, g_{+}\left(\bar{\psi}\left(A d_{g_{-}^{-1}}^{*} \psi\left(X_{+}\right)\right)\right) g_{-}\right\rangle \\
& -\left\langle\eta,\left[g_{+}\left(\bar{\psi}\left(A d_{g_{-}^{-1}}^{*} \psi\left(X_{+}\right)\right)\right) g_{-}, g_{+}\left(\bar{\psi}\left(A d_{g_{-}^{-1}}^{*} \psi\left(Y_{+}\right)\right)\right) g_{-}\right]\right\rangle
\end{aligned}
$$

showing that $\omega_{c} \mid \mathcal{N}\left(g_{-}, \eta_{-}\right)=-d \Theta$ as stated.

Therefore, the Lagrangian function on $\mathcal{N}\left(g_{-}, \eta_{-}\right)$is

$$
L_{\mathcal{N}\left(g_{-}, \eta_{-}\right)}(g, \dot{g})=\left\langle\eta, g^{-1} \dot{g}\right\rangle-\left.\mathcal{H}(g, \eta)\right|_{\mathcal{N}\left(g_{-}, \eta_{-}\right)},
$$

where $\left.\mathcal{H}(g, \eta)\right|_{\mathcal{N}\left(g_{-}, \eta_{-}\right)}$is the restriction of $\mathcal{H}(g, \eta)$ to $\mathcal{N}\left(g_{-}, \eta_{-}\right)$, with

$$
\mathcal{H}(g, \eta)=\frac{1}{2}\left(\bar{\psi}\left(J_{B}^{L \wedge}(g, \eta)\right), \mathcal{E} \bar{\psi}\left(J_{B}^{L \wedge}(g, \eta)\right)\right)_{\mathfrak{g}^{\wedge}},
$$

where, having in mind that $\left(g_{-}, \eta_{-}\right) \in \operatorname{ker} C \times \operatorname{Char}\left(\mathfrak{g}_{-}\right)$,

$$
\left.J_{B}^{L \wedge}(g, \eta)\right|_{\mathcal{N}\left(g_{-}, \eta_{-}\right)}=\left(\operatorname{Ad}_{g^{-1}}^{G *} \eta+C\left(g_{+}\right), 1\right) .
$$

Then

$$
\begin{aligned}
H\left(g_{+}, \eta_{+}\right): & =\left.\mathcal{H}(g, \eta)\right|_{\mathcal{N}\left(g_{-}, \eta_{-}\right)} \\
= & \frac{1}{2}\left(\bar{\psi}\left(\operatorname{Ad}_{g^{-1}}^{G *} \eta+C\left(g_{+}\right)\right), \mathcal{E} \bar{\psi}\left(\operatorname{Ad}_{g^{-1}}^{G *} \eta+C\left(g_{+}\right)\right)\right)_{\mathfrak{g}^{\wedge}} .
\end{aligned}
$$


On the other hand, on $\mathcal{N}\left(g_{-}, \eta_{-}\right)$we have that

$$
g^{-1} \dot{g}=g_{-}^{-1} g_{+}^{-1} \dot{g}_{+} g_{-}=A d_{g_{-}^{-1}}^{G}\left(g_{+}^{-1} \dot{g}_{+}\right)
$$

therefore

$$
\begin{aligned}
& L_{\mathcal{N}}(g, \dot{g}) \\
= & \left\langle\eta, \operatorname{Ad}_{g_{-}^{-1}}^{G}\left(g_{+}^{-1} \dot{g}_{+}\right)\right\rangle-\frac{1}{2}\left(\bar{\psi}\left(\operatorname{Ad}_{g^{-1}}^{G *} \eta+C\left(g_{+}\right)\right), \mathcal{E} \bar{\psi}\left(\operatorname{Ad}_{g^{-1}}^{G *} \eta+C\left(g_{+}\right)\right)\right)_{\mathfrak{g}},
\end{aligned}
$$

which is equivalent to

$$
\begin{aligned}
& L_{\mathcal{N}}(g, \dot{g}) \\
= & \left(\bar{\psi}\left(\operatorname{Ad}_{g_{-}^{-1}}^{G *} \eta\right), g_{+}^{-1} \dot{g}_{+}\right)_{\mathfrak{g}}-\frac{1}{2}\left(\bar{\psi}\left(\operatorname{Ad}_{g_{-}^{-1}}^{G *} \eta\right), \mathcal{E}_{g_{+}} \bar{\psi}\left(\operatorname{Ad}_{g_{-}^{-1}}^{G *} \eta\right)\right)_{\mathfrak{g}} \\
& +\left(\bar{\psi}\left(\operatorname{Ad}_{g_{-}^{-1}}^{G *} \eta\right), \mathcal{E}_{g_{+}} \bar{\psi}\left(C\left(g_{+}^{-1}\right)\right)\right)_{\mathfrak{g}}-\frac{1}{2}\left(\bar{\psi}\left(C\left(g_{+}^{-1}\right)\right), \mathcal{E}_{g_{+}} \bar{\psi}\left(C\left(g_{+}^{-1}\right)\right)\right)_{\mathfrak{g}} .
\end{aligned}
$$

By replacing the fiber coordinate $\eta_{+}$as a function of the velocity, as it was obtained from the first Hamilton equation in Eq. (13),

$$
A d_{g_{-}^{-1}}^{G *} \eta_{+}=\psi\left(\mathcal{G}_{g_{+}} g_{+}^{-1} \dot{g}_{+}-\mathcal{B}_{g_{+}} \bar{\psi}\left(C\left(g_{+}^{-1}\right)\right)+\left(\mathcal{B}_{g_{+}}-\Pi_{\mathfrak{g}_{-}} A d_{g_{-}}^{G}\right) \bar{\psi}\left(\eta_{-}\right)\right)
$$

we get

$$
\begin{aligned}
L_{\mathcal{N}}(g, \dot{g})= & \frac{1}{2}\left(\mathcal{G}_{g_{+}} g_{+}^{-1} \dot{g}_{+}, g_{+}^{-1} \dot{g}_{+}\right)_{\mathfrak{g}}-\left(g_{+}^{-1} \dot{g}_{+}, \mathcal{B}_{g} \bar{\psi}\left(C\left(g_{+}^{-1}\right)-\eta_{-}\right)\right)_{\mathfrak{g}} \\
& -\frac{1}{2}\left(\bar{\psi}\left(C\left(g_{+}^{-1}\right)-\eta_{-}\right), \mathcal{G}_{g} \bar{\psi}\left(C\left(g_{+}^{-1}\right)-\eta_{-}\right)\right)_{\mathfrak{g}} .
\end{aligned}
$$

Finally, it can also be written as the Poisson-Lie $\sigma$-model:

$$
L_{\mathcal{N}}(g, \dot{g})=\frac{1}{2}\left(\mathcal{R}_{g_{+}}^{+}\left(g_{+}^{-1} \dot{g}_{+}-\bar{\psi}\left(C\left(g_{+}^{-1}\right)-\eta_{-}\right)\right),\left(g_{+}^{-1} \dot{g}_{+}+\bar{\psi}\left(C\left(g_{+}^{-1}\right)-\eta_{-}\right)\right)\right)_{\mathfrak{g}},
$$

where $\mathcal{R}_{g_{+}}^{ \pm}:=\mathcal{B}_{g_{+}} \pm \mathcal{G}_{g_{+}}$

Thus, we have shown that the collective Hamiltonian system (15) is the phase space version of the Lagrangian system introduced in the context on the Poisson-Lie T-duality in Ref. 15. Amazingly, it arises through the Dirac restriction method of the larger phase $G \times \mathfrak{g}^{*}$ of a noncollective non-Adinvariant Hamiltonian function.

\section{Centrally extended loop algebras}

Let $\mathfrak{g}$ be the loop algebra $\mathfrak{g}=\mathrm{Lh}$ for some Lie algebra $\mathfrak{h}$ equipped with the bilinear form

$$
(X, Y)_{\mathfrak{g}}=\frac{1}{2 \pi} \int_{S^{1}}(X(s), Y(s))_{\mathfrak{h}} d s .
$$

Then we define the coadjoint cocycle $C_{\mathrm{k}}: H \rightarrow \mathfrak{h}^{*}$,

$$
C_{\mathrm{k}}(g)=\mathrm{k} \psi\left(g^{\prime} g^{-1}\right)
$$

which satisfies

$$
C_{\mathrm{k}}(g h)=\operatorname{Ad}_{g-1}^{G *} C_{\mathrm{k}}(h)+C_{\mathrm{k}}(g) .
$$

It extends to a cocycle on $G=L H$ by the identification of the dual $\mathfrak{g}^{*}$ through the bilinear form defined above, in such a way that

$$
\left\langle C_{\mathrm{k}}(g), Y\right\rangle=\frac{\mathrm{k}}{2 \pi} \int_{S^{1}}\left(g^{\prime} g^{-1}, Y\right)_{\mathfrak{h}} d s
$$


so we define $c_{\mathrm{k}}: \mathfrak{g} \otimes \mathfrak{g} \longrightarrow \mathbb{R}$ as

$$
c_{\mathrm{k}}(X, Y)=\frac{\mathrm{k}}{2 \pi} \int_{S^{1}}\left(X(s), Y^{\prime}(s)\right)_{\mathfrak{h}} d s
$$

with the map $\hat{c}_{\mathrm{k}}: \mathfrak{g} \longrightarrow \mathfrak{g}^{*}$,

$$
\hat{c}_{\mathrm{k}}(X)=-\mathrm{k} \psi\left(X^{\prime}\right) .
$$

Let us substitute cocycles (17) and (18) in the Lagrange equation (14), to obtain

$$
\begin{aligned}
& \frac{\partial}{\partial t}\left(\mathcal{G}_{g_{+}} g_{+}^{-1} \dot{g}_{+}+\mathrm{k} \mathcal{B}_{g_{+}} g_{+}^{-1} g_{+}^{\prime}+\mathcal{B}_{g_{+}} \bar{\psi}\left(\eta_{-}\right)+\bar{\psi}\left(\eta_{-}\right)\right) \\
& -\mathrm{k} \frac{\partial}{\partial x}\left(\mathcal{B}_{g_{+}} g_{+}^{-1} \dot{g}_{+}+\mathrm{k} \mathcal{G}_{g_{+}} g_{+}^{-1} g_{+}^{\prime}+\mathcal{G}_{g_{+}} \bar{\psi}\left(\eta_{-}\right)\right) \\
& =\Pi_{\mathfrak{g}_{-}}\left[\mathcal{B}_{g_{+}} g_{+}^{-1} \dot{g}_{+}+\mathcal{G}_{g_{+}}\left(\mathrm{k}_{+}^{-1} g_{+}^{\prime}+\bar{\psi}\left(\eta_{-}\right)\right), \mathcal{G}_{g_{+}} g_{+}^{-1} \dot{g}_{+}+\mathcal{B}_{g_{+}}\left(\mathrm{kg}_{+}^{-1} g_{+}^{\prime}+\bar{\psi}\left(\eta_{-}\right)\right)+\bar{\psi}\left(\eta_{-}\right)\right] .
\end{aligned}
$$

In this case, and introducing $\partial_{ \pm}=\frac{\partial}{\partial t} \pm \mathrm{k} \frac{\partial}{\partial x}$, the Lagrangian density (16) turns in to

$$
\mathcal{L}_{\mathcal{N}}\left(g_{+}\right)=\frac{1}{2}\left(\mathcal{R}_{g_{+}}\left(g_{+}^{-1} \partial_{+} g_{+}+\bar{\psi}\left(\eta_{-}\right)\right),\left(g_{+}^{-1} \partial_{-} g_{+}-\bar{\psi}\left(\eta_{-}\right)\right)\right)_{\mathfrak{g}} .
$$

Following, ${ }^{17}$ we use the decomposition of the Lie algebra $\mathfrak{g}$ as the direct sum of the eingenspaces of the operator $\mathcal{E}_{g_{+}}$in order to eliminate the $g_{+}$-dependence in the operator $\mathcal{R}_{g_{+}}$, so we obtain a purely world sheet depending operator. Since $\left(\left(\mathcal{R}_{e}^{ \pm}\right)^{-1} X_{-}, X_{-}\right) \in \mathcal{E}^{ \pm}(e), \operatorname{Ad}_{g_{+}^{-1}}^{G}\left(\left(\mathcal{R}_{e}^{ \pm}\right)^{-1} X_{-}, X_{-}\right) \in$ $\mathcal{E}^{ \pm}(g)$, which implies the relation

$$
\operatorname{Ad}_{g_{+}}^{G}\left(\mathcal{R}_{g}^{ \pm}\right)^{-1} \Pi_{\mathfrak{g}_{-}} \operatorname{Ad}_{g_{+}^{-1}}^{G} X_{-}=\left(\mathcal{R}_{e}^{ \pm}\right)^{-1} X_{-}+\Pi_{\mathfrak{g}_{+}} \operatorname{Ad}_{g_{+}}^{G} \Pi_{\mathfrak{g}_{+}} \operatorname{Ad}_{g_{+}^{-1}}^{G} X_{-} .
$$

By using the right translated Poisson-Lie bivector on $G_{+}, \pi_{+}^{R}: G_{+} \longrightarrow \mathfrak{g}_{+} \otimes \mathfrak{g}_{+}$is defined from the relation $^{16}$

$$
\left\langle\psi\left(X_{-}^{\prime}\right) \otimes \psi\left(X_{-}^{\prime \prime}\right), \pi_{+}^{R}\left(g_{+}\right)\right\rangle:=\left(\Pi_{-} \mathrm{Ad}_{g_{+}^{-1}}^{G} X_{-}^{\prime}, \Pi_{+} \mathrm{Ad}_{g_{+}^{-1}}^{G} X_{-}^{\prime \prime}\right)_{\mathfrak{g}}
$$

and regarding it as the linear map $\pi_{+}^{R}\left(g_{+}\right): \mathfrak{g}_{-} \longrightarrow \mathfrak{g}_{+}$such that

$$
\left(\pi_{+}^{R}\left(g_{+}\right) X_{-}, Y_{-}\right)_{\mathfrak{g}}:=\left\langle\psi\left(Y_{-}\right) \otimes \psi\left(X_{-}\right), \pi_{+}^{R}\left(g_{+}\right)\right\rangle,
$$

$\forall Y_{-} \in \mathfrak{g}_{-}$, we identify

$$
\pi_{+}^{R}\left(g_{+}\right)=-\Pi_{\mathfrak{g}_{+}} \operatorname{Ad}_{g_{+}}^{G} \Pi_{\mathfrak{g}_{+}} \operatorname{Ad}_{g_{+}^{-1}}^{G} \Pi_{-} .
$$

So, the above relation turns in to

$$
\operatorname{Ad}_{g_{+}}^{G}\left(\mathcal{R}_{g}^{ \pm}\right)^{-1} \Pi_{\mathfrak{g}_{-}} \operatorname{Ad}_{g_{+}^{-1}}^{G} \Pi_{\mathfrak{g}_{-}}=\left(\left(\mathcal{R}_{e}^{ \pm}\right)^{-1}-\pi_{+}^{R}\left(g_{+}\right)\right) \Pi_{\mathfrak{g}_{-}},
$$

which allows us to substitute $\mathcal{R}_{g}^{ \pm}$into the Lagrangian density (19) to get

$$
\mathcal{L}_{\mathcal{N}}(g)=\frac{1}{2}\left(\left(\left(\mathcal{R}_{e}^{ \pm}\right)^{-1}-\pi_{+}^{R}\left(g_{+}\right)\right)^{-1}\left(g_{+}^{-1} \partial_{+} g_{+}+\bar{\psi}\left(\eta_{-}\right)\right),\left(g_{+}^{-1} \partial_{-} g_{+}-\bar{\psi}\left(\eta_{-}\right)\right)\right)_{\mathfrak{g}},
$$

which coincides with the T-dual sigma model on the target $G_{+}$introduced by Klimcik and Severa. ${ }^{15}$

\section{CONCLUSIONS}

We studied the restriction to a family of submanifolds of the second class constraint type in the cotangent bundle of a double Poisson-Lie group, equipped with a cocycle extended symplectic form. For a given Hamiltonian system on this phase space, it is this extension that gives rise to the WZtopological term in the corresponding action. Then we built up the corresponding Dirac brackets showing that, for the usual 2-cocycle in loop groups, it exhibits no contribution from it. It is in this sense that we say that a WZNW phase space restricts to a $\sigma$-model one. Looking for symmetries 
on the constrained phase spaces, we worked out the left translation action although it is manifestly not a symmetry in the whole space because the 2-cocycle is defined on right invariant vector fields. However, we have shown that in the fiber spaces on points $\left(g_{-}, \eta_{-}\right) \in \operatorname{ker} C \times \operatorname{Char}\left(\mathfrak{g}_{-}\right)$, the left translation momentum function closes a Lie algebra under the corresponding Dirac bracket, so the left translation symmetry becomes restored.

This facts suggested the study of collective dynamical systems on these phase subspaces, which in some way resembles the situation in the Hamiltonian approach WZNW model, see Ref. 14, where the Marsden-Weinstein reduction procedure has a first stage of reduction in relation to the obvious right translation symmetry. However, there still remains a residual left translation symmetry that, once reduced, leads to the chiral mode solutions.

Thus we studied a Hamiltonian system on $G \times \mathfrak{g}^{*}$ ruled by a Hamilton function of the momentum maps of left translation on the constrained submanifolds, regarded as functions on the whole space. This Hamiltonian turns collective when restricted to $\mathcal{N}\left(g_{-}, \eta_{-}\right)$, and leads to nice dynamics flowing along orbits of some curves in group $G$ through the action found in (8). Amazingly, these restricted Hamiltonian systems turn to be Poisson-Lie $\sigma$-models, with some additional terms involving the parameter $\eta_{-}$of the fiber. In summary, starting from a WZNW type model, through a restriction procedure implemented by the Dirac method we arrived at the Poisson-Lie $\sigma$-models.

Most of the issues developed here are much involved with the Hamiltonian framework for the Poisson-Lie T-duality, as is clear from the restriction of the left translation symmetry to the special fibers. The T-dual side can be obtained, with some subtleties, by considering the fibration $G \times \mathfrak{g}^{*} \longrightarrow G_{+} \times \mathfrak{g}_{+}^{*}$ as a starting point, and repeating the above construction.

Although the current work is purely classical, this formulation as a second class constrained system allows one to address the quantization in the scheme of the functional integral as a constrained system defined on the whole $G \times \mathfrak{g}^{*}$.

\section{ACKNOWLEDGMENTS}

H.M. thanks CONICET, Argentina, for financial support.

${ }^{1}$ M. Abraham and J. Marsden, Foundations of Mechanics, 2nd ed. (Benjamin/Cummings, Reading, MA, 1978).

${ }^{2}$ A. Yu. Alekseev, P. Schaller, and T. Strobl, "The topological G/G WZW model in the generalized momentum representation,” Phys. Rev. D 52, 7146-7160 (1995).

${ }^{3}$ V. I. Arnold, "Sur la géométrie diffé rentielle des groupes de Lie de dimension infinie et ses applications à l'hydrodynamique des fluides parfaites," Annl. l'instit. Fourier, Tome 16(1), 319-361 (1966); Mathematical Methods of Classical Mechanics (Springer-Verlag, New York, 1989).

${ }^{4}$ V. I. Arnold and A. B. Givental, Symplectic Geometry, in Dynamical Systems IV (Encyclopaedia of Mathematical Sciences), edited by V. I. Arnold and S. P. Novikov (Springer, 1993).

${ }^{5}$ I. Calvo, F. Falceto, and D. García-Álvarez, “Topological Poisson sigma models on Poisson-Lie groups,” J. High Energy Phys. 10 (2003) 033.

${ }^{6}$ A. Cabrera and H. Montani, "Hamiltonian loop group actions and T-Duality for group manifolds," J. Geom. Phys. 56, $1116-1143$ (2006).

${ }^{7}$ A. Cabrera, H. Montani, and M. Zuccalli, “Poisson Lie T-duality and nontrivial monodromies," J. Geom. Phys. 59, 576-599 (2009).

${ }^{8}$ S. Capriotti and H. Montani, "Dirac method and symplectic submanifolds in the cotangent bundle of a factorizable Lie group," J. Math. Phys. 52, 073504 (2011).

${ }^{9}$ P. A. M. Dirac, Lectures on Quantum Mechanics, Belfer Graduate School of Science Monographs Series Number 2 (Belfer Graduate School of Science, Yeshiva University, New York, 1964).

${ }^{10}$ V. G. Drinfeld, "Hamiltonian structures on Lie groups, Lie algebras and the geometric meaning of the classical Yang-Baxter equation," Soviet Math. Dokl. 27, 68-71 (1983).

${ }^{11}$ L. D. Faddeev and L. A. Takhtajan, Hamiltonian Method in the Theory of Solitons (Springer-Verlag, Heidelberg, 1987).

${ }^{12}$ F. Falceto and K. Gawedzki, "Boundary G/G theory and topological Poisson-Lie sigma model," Lett. Math. Phys. 59, 61-79 (2002).

${ }^{13}$ V. Guillemin and S. Sternberg, Symplectic Techniques in Physics (Cambridge University Press, Cambridge, 1984).

${ }^{14}$ J. Harnad, "Constrained Hamiltonian systems on Lie groups, moment map reductions and central extensions," Can. J. Phys. 72, 375-388 (1994); J. Harnad and B. A. Kupershmidt, "Symplectic Geometries on $T^{*} \widetilde{G}$, Hamiltonian group actions and integrable systems," J. Geom. Phys. 16, 168-206 (1995).

${ }^{15}$ C. Klimcik and P. Severa, "Poisson-Lie T-duality and loop groups of Drinfeld doubles," Phys. Lett. B 351, 455-462 (1995); "Dual non-Abelian duality and the Drinfeld double," ibid. 372, 65-71 (1996); C. Klimcik, "Poisson-Lie T-duality," Nucl. Phys. Proc. Suppl. 46, 116-121 (1996). 
${ }^{16}$ J.-H. Lu and A. Weinstein, "Poisson Lie groups, dressing transformations and Bruhat decompositions," J. Differential Geom. 31, 501-526 (1990).

${ }^{17}$ S. Majid and E. J. Beggs, "Poisson-Lie T-duality for quasitriangular Lie bialgebras," Commun. Math. Phys. 220, 455-488 (2001).

${ }^{18} \mathrm{~J}$. Marsden and T. Ratiu, Introduction to Mechanics and Symmetry, 2nd ed. (Benjamin/Cummings, Reading, MA, 1978).

${ }^{19}$ J. E. Marsden and A. Weinstein, "Reduction of symplectic manifolds with symmetry," Rep. Math. Phys. 5, 121-131 (1974).

${ }^{20}$ A. Pressley and G. Segal, Loop Groups (Clarendon Press, Oxford, 1986).

${ }^{21}$ A. G. Reyman and M. A. Semenov-Tian-Shansky, Group-Theoretical Methods in the Theory of Finite-Dimensional Integrable Systems, in Dynamical Systems VII (Encyclopaedia of Mathematical Sciences), edited by V. I. Arnold and S. P. Novikov (Springer, 1993).

${ }^{22}$ M. A. Semenov-Tian-Shansky, "Dressing transformations and Poisson group actions," Publ. RIMS, Kyoto Univ. 21, $1237-1260(1985)$ 